\title{
An explicit marching-on-in-time scheme for solving the time domain Kirchhoff integral equation
}

Rui Chen, Sadeed Bin Sayed, Noha Alharthi, David Keyes, and Hakan Bagci

Citation: The Journal of the Acoustical Society of America 146, 2068 (2019); doi: 10.1121/1.5125259

View online: https://doi.org/10.1121/1.5125259

View Table of Contents: https://asa.scitation.org/toc/jas/146/3

Published by the Acoustical Society of America

\section{ARTICLES YOU MAY BE INTERESTED IN}

Source triangulation utilizing three-dimensional arrivals: Application to the search for the ARA San Juan submarine

The Journal of the Acoustical Society of America 146, 2104 (2019); https://doi.org/10.1121/1.5125251

Method of numerical Green's function determination for far-field scattering solutions from objects at a watersediment interface

The Journal of the Acoustical Society of America 146, 2093 (2019); https://doi.org/10.1121/1.5125250

Efficient, wide-band rigid-body and elastic scattering computations using transient equivalent sources

The Journal of the Acoustical Society of America 146, 2080 (2019); https://doi.org/10.1121/1.5125424

Beam tracing for two- and three-dimensional problems in ocean acoustics

The Journal of the Acoustical Society of America 146, 2016 (2019); https://doi.org/10.1121/1.5125262

$3 \mathrm{D}$ acoustic propagation through an estuarine salt wedge at low-to-mid-frequencies: Modeling and measurement The Journal of the Acoustical Society of America 146, 1888 (2019); https://doi.org/10.1121/1.5125258

Measurements and modeling of acoustic propagation in a scale model canyon

The Journal of the Acoustical Society of America 146, 1858 (2019); https://doi.org/10.1121/1.5125130

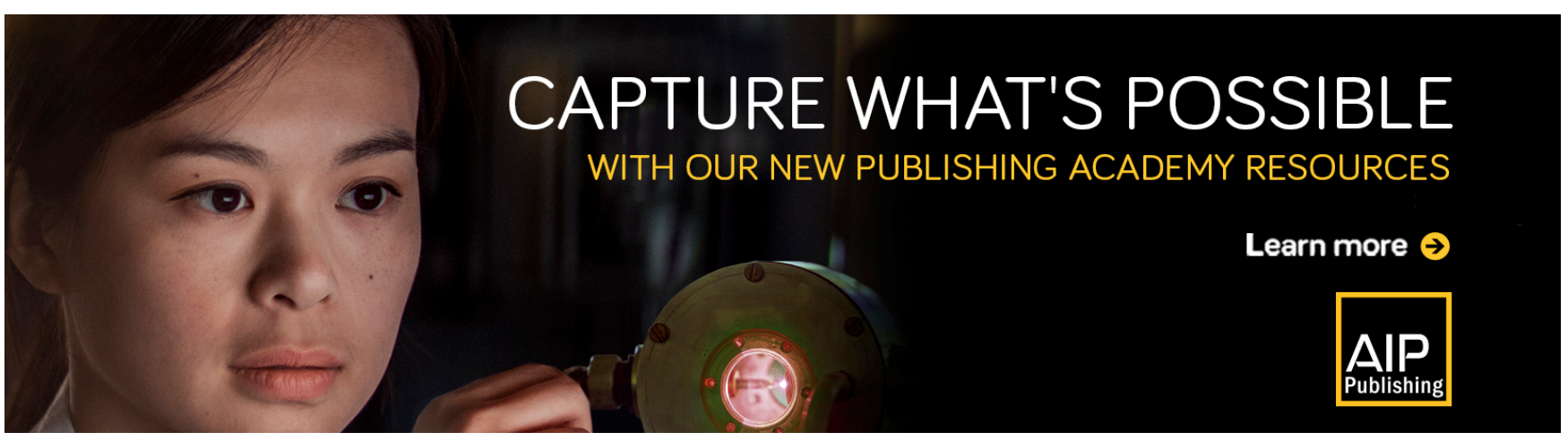




\title{
An explicit marching-on-in-time scheme for solving the time domain Kirchhoff integral equation
}

\author{
Rui Chen, ${ }^{1, \text { a) }}$ Sadeed Bin Sayed, ${ }^{1}$ Noha Alharthi, ${ }^{2, b)}$ David Keyes, ${ }^{2, b)}$ and Hakan Bagci ${ }^{1}$ \\ ${ }^{1}$ Division of Computer, Electrical, and Mathematical Science and Engineering, 4700 King Abdullah \\ University of Science and Technology, Thuwal 23955-6900, Saudi Arabia \\ ${ }^{2}$ Extreme Computing Research Center, 4700 King Abdullah University of Science and Technology, \\ Thuwal 23955-6900, Saudi Arabia
}

(Received 31 December 2018; revised 24 April 2019; accepted 28 May 2019; published online 1 October 2019)

\begin{abstract}
A fully explicit marching-on-in-time (MOT) scheme for solving the time domain Kirchhoff (surface) integral equation to analyze transient acoustic scattering from rigid objects is presented. A higherorder Nyström method and a $\mathrm{PE}(\mathrm{CE})^{m}$-type ordinary differential equation integrator are used for spatial discretization and time marching, respectively. The resulting MOT scheme uses the same time step size as its implicit counterpart (which also uses Nyström method in space) without sacrificing from the accuracy and stability of the solution. Numerical results demonstrate the accuracy, efficiency, and applicability of the proposed explicit MOT solver. ( 2019 Author(s). All article content, except where otherwise noted, is licensed under a Creative Commons Attribution (CC BY) license (http://creativecommons.org/licenses/by/4.0/). https://doi.org/10.1121/1.5125259
\end{abstract}

[MP]

Pages: 2068-2079

\section{INTRODUCTION}

Transient acoustic scattering from rigid objects can be numerically analyzed using either differential equation solv$\mathrm{ers}^{1-4}$ or time domain surface integral equation (TDSIE) solvers. ${ }^{5-14}$ TDSIE solvers have several advantages over differential equation solvers. ${ }^{4}$ (i) They require only a twodimensional discretization of the scatterer surface as opposed to a three-dimensional discretization of the whole computation domain. (ii) They are free from numerical dispersion since they do not discretize spatial/temporal derivatives using finite differences or finite elements. (iii) They do not need approximate absorbing boundary conditions to truncate the unbounded physical domain into the computation domain.

To construct a TDSIE, the scattered pressure field is expressed as a spatio-temporal convolution of the velocity potential generated on the surface of the scatterer (upon excitation) and the Green function of the unbounded background medium. Then, a TDSIE in (unknown) velocity potential is obtained by enforcing the boundary condition of the total pressure field (excitation plus the scattered pressure field) on the rigid surface of the scatterer. One of the classical schemes for solving TDSIEs is the marching-on-in-time (MOT) method. ${ }^{5,8-11,13,14}$ The MOT-TDSIE solvers expand the unknown velocity potential in terms of (local) spatial and temporal basis functions. Inserting this expansion into the TDSIE and testing the resulting equation in space and time yields system of equations. This system of equations is solved/updated for the unknown expansion coefficients through marching in time. The right-hand side of this system consists of the tested excitation and the scattered

\footnotetext{
${ }^{a}$ Electronic mail: rui.chen@kaust.edu.sa

b) Also at: Division of Computer, Electrical, and Mathematical Science and Engineering, 4700 King Abdullah University of Science and Technology, Thuwal 23955-6900, Saudi Arabia.
}

pressure field expressed as a discretized spatio-temporal convolution of the Green function and history of the velocity potential.

Despite the advantages of the TDSIE solvers listed in the first paragraph, use of the MOT-TDSIE solvers in analyzing transient acoustic scattering has been rather limited. This can be attributed to the difficulty of obtaining a stable solution as well as the high cost of computing the discretized spatio-temporal convolution. The former bottleneck has been alleviated with the development of new temporal basis functions ${ }^{15}$ and testing schemes, ${ }^{9,16,17}$ as well as accurate integration methods. ${ }^{17-20}$ To reduce the high computational cost, plane wave time domain (PWTD) algorithm ${ }^{11,12,21,22}$ and the fast Fourier transform (FFT)-based acceleration schemes ${ }^{23-26}$ have been developed.

An MOT solver can be either explicit ${ }^{13,14}$ or implicit ${ }^{5-7}$ depending on the types of (spatial and temporal) basis functions and the testing scheme, as well as the time step size. The implicit MOT schemes are more stable and permit larger time steps without sacrificing from stability. But they call for solution of a matrix equation at every time step. On the other hand, the explicit MOT schemes do not require a matrix equation solution, however, they have to use a smaller time step size to ensure the stability. To overcome this drawback, a quasi-explicit MOT scheme has been developed for solving the time domain magnetic field integral equation (TDMFIE) of electromagnetics. ${ }^{27}$ This scheme employs RaoWilton-Glisson (RWG) functions ${ }^{28}$ for spatial discretization and can use time step sizes as large as its implicit counterpart while maintaining the stability and the accuracy of the solution. However, it still needs solution of a sparse but unstructured (Gram) matrix equation at every time step because the RWG basis functions have overlapping supports/domains.

In this work, relying on the idea behind this quasiexplicit MOT scheme, a fully explicit MOT scheme is 
formulated and implemented to solve the time domain Kirchhoff (surface) integral equation (TDKIE) ${ }^{10,29}$ for analysis of transient acoustic scattering from rigid objects. The TDKIE is cast in the form of a first-order ordinary differential equation (ODE) in time. The unknown velocity potential generated on the surface of the scatterer is expanded in terms of higher-order polynomials in space with time dependent (unknown) coefficients. Inserting this expansion into the TDKIE and point testing the resulting equation in space yield a system of ODEs (i.e., Nyström method in space). This ODE system is then integrated in time using a $\mathrm{PE}(\mathrm{CE})^{m}$ scheme for the (samples of) the unknown expansion coefficients. The resulting MOT scheme does not call for a matrix equation solution since the Gram matrix (resulting from the point testing of the higher-order polynomials) is diagonal. This explicit MOT scheme uses the same time step size as its implicit counterpart (which also uses Nyström method in space) without sacrificing from the stability and the accuracy of the solution. Furthermore, it is faster than the implicit MOT scheme under low frequency excitation (i.e., for large time step sizes) since the matrix equation solved by the implicit scheme becomes fuller with the increasing time step size. Indeed, numerical results demonstrate the accuracy, efficiency, and applicability of the proposed explicit MOT solver.

The remainder of the paper is organized as follows. Section II A briefly formulates the TDKIE. Section II B provides the higher-oder spatial and temporal expansions used for representing the unknown velocity potential. Sections II C and II D detail the implicit and explicit MOT schemes, respectively, and Sec. II E compares the computational complexities of these two schemes under high and lowfrequency excitations (for small and large time steps). Sections III A and III B provide numerical results that demonstrate the accuracy and efficiency, and the applicability of the proposed explicit solver, respectively. Finally, Sec. IV summarizes the work detailed in the paper and outlines future research directions.

\section{FORMULATION}

\section{A. TDKIE}

Let $S$ represent the surface of an acoustic scatterer object residing in an unbounded homogeneous background medium with fluid density $\rho_{0}$ and wave speed $c_{0}$. An acoustic wave with velocity potential $\varphi^{\mathrm{i}}(\mathbf{r}, t)$ and pressure field $p^{\mathrm{i}}(\mathbf{r}, t)=-\rho_{0} \partial_{t} \varphi^{\mathrm{i}}(\mathbf{r}, t)$ is incident on $S$; it is assumed that $\varphi^{\mathrm{i}}(\mathbf{r}, t)$ and $p^{\mathrm{i}}(\mathbf{r}, t)$ are band-limited and vanishingly small for $t \leq 0, \forall \mathbf{r} \in S$. Let $\varphi^{\mathrm{s}}(\mathbf{r}, t)$ and $p^{\mathrm{s}}(\mathbf{r}, t)=-\rho_{0} \partial_{t} \varphi^{\mathrm{s}}(\mathbf{r}, t)$ represent the velocity potential and pressure field of the wave scattered from $S$. The total velocity potential is the sum of $\varphi^{\mathrm{i}}(\mathbf{r}, t)$ and $\varphi^{\mathrm{s}}(\mathbf{r}, t): \varphi(\mathbf{r}, t)=\varphi^{\mathrm{i}}(\mathbf{r}, t)+\varphi^{\mathrm{s}}(\mathbf{r}, t)$. Similarly, total pressure field is the sum of $p^{\mathrm{i}}(\mathbf{r}, t)$ and $p^{\mathrm{s}}(\mathbf{r}, t): \quad p(\mathbf{r}, t)=p^{\mathrm{i}}(\mathbf{r}, t)+p^{\mathrm{s}}(\mathbf{r}, t)$. One can obtain the TDKIE in (unknown) $\varphi(\mathbf{r}, t)$ for the exterior problem (in the background medium, outside the volume enclosed by $S$ ) using the Kirchhoff-Helmholtz theorem, ${ }^{30}$

$$
\begin{aligned}
\partial_{t} \varphi^{\mathrm{i}}(\mathbf{r}, t)= & \frac{1}{2} \partial_{t} \varphi(\mathbf{r}, t)-\partial_{t} \int_{S} \varphi\left(\mathbf{r}^{\prime}, t\right) * \hat{\mathbf{n}}^{\prime}\left(\mathbf{r}^{\prime}\right) \cdot \nabla^{\prime} G(R, t) d s^{\prime} \\
& +\partial_{t} \int_{S} \hat{\mathbf{n}}^{\prime}\left(\mathbf{r}^{\prime}\right) \cdot \nabla^{\prime} \varphi\left(\mathbf{r}^{\prime}, t\right) * G(R, t) d s^{\prime}, \quad \mathbf{r} \in S
\end{aligned}
$$

Here, $G(R, t)=\delta\left(t-R / c_{0}\right) /(4 \pi R)$ is the Green function in the background medium, $R=\left|\mathbf{r}-\mathbf{r}^{\prime}\right|$ is the distance between the observation point $\mathbf{r}$ and the source point $\mathbf{r}^{\prime}, \delta(\cdot)$ is the Dirac impulse function, and $\hat{\mathbf{n}}^{\prime}\left(\mathbf{r}^{\prime}\right)$ is the outward pointing unit normal to $S$ at $\mathbf{r}^{\prime}$, and $\partial_{t}$ and * denote the temporal derivative and convolution, respectively. Note that the first integral on the left hand side of Eq. (1) should be evaluated in the principal value sense. For an acoustically rigid object, the normal of the (total) velocity vanishes, ${ }^{30}$ i.e., $\varphi(\mathbf{r}, t)$ satisfies the boundary condition

$$
\hat{\mathbf{n}}(\mathbf{r}) \cdot \nabla \varphi(\mathbf{r}, t)=0, \quad \mathbf{r} \in S,
$$

where $\hat{\mathbf{n}}(\mathbf{r})$ is the outward pointing unit normal to $S$ at $\mathbf{r}$. Inserting Eq. (2) into Eq. (1) yields the TDKIE for a rigid object, ${ }^{10,30}$

$$
\begin{aligned}
& \frac{1}{2} \partial_{t} \varphi(\mathbf{r}, t)-\partial_{t} \int_{S} \varphi\left(\mathbf{r}^{\prime}, t\right) * \hat{\mathbf{n}}^{\prime}\left(\mathbf{r}^{\prime}\right) \cdot \nabla^{\prime} G(R, t) d s^{\prime} \\
& \quad=\partial_{t} \varphi^{\mathrm{i}}(\mathbf{r}, t) .
\end{aligned}
$$

\section{B. Higher-order spatial representation}

To solve Eq. (3) numerically, $S$ is discretized into $N_{\mathrm{p}}$ curvilinear triangular patches, and $\varphi(\mathbf{r}, t)$ is spatially expanded/approximated using higher-order interpolation functions as ${ }^{31-33}$

$$
\varphi(\mathbf{r}, t)=\sum_{p=1}^{N_{\mathrm{p}}} \sum_{i=1}^{N_{\mathrm{u}}}\{\mathbf{I}(t)\}_{(i, p)} \vartheta^{-1}(\mathbf{r}) L_{(i, p)}(\mathbf{r}) .
$$

Here, $N_{\mathrm{u}}$ is the number of interpolation points on each patch, $L_{(i, p)}(\mathbf{r})$ is the Lagrange interpolation function defined at $\mathbf{r}_{(i, p)}\left(i\right.$ th interpolation point on $p$ th patch), ${ }^{33}$ and $\{\mathbf{I}(t)\}_{(i, p)}$ is the time-dependent unknown expansion coefficient at $\mathbf{r}_{(i, p)}$. In Eq. (4),

$$
\vartheta(\mathbf{r})=\sqrt{\left(\partial_{\xi} \mathbf{r} \cdot \partial_{\xi} \mathbf{r}\right)\left(\partial_{\eta} \mathbf{r} \cdot \partial_{\eta} \mathbf{r}\right)-\left(\partial_{\xi} \mathbf{r} \cdot \partial_{\eta} \mathbf{r}\right)^{2}}
$$

is the Jacobian of the coordinate transformation between the Cartesian coordinate system and a two-dimensional space with variables $\xi$ and $\eta$. Here, derivatives $\partial_{\xi}$ and $\partial_{\eta}$ should be applied separately to the components of the threedimensional vector $\mathbf{r}$. This quadratic transformation enables the description of the curvilinear triangular patch (defined by three nodes at its vertices and another set of three nodes at the mid-points of its edges) [Fig. 1(a)] using a (flat) rightangle triangular patch generated in the $(\xi, \eta)$ space [Fig. 1(b)]. ${ }^{3}$ Figures 1(a) and 1(b) also show the interpolation points $\mathbf{r}_{(i, p)}$ in the Cartesian coordinate system and their 


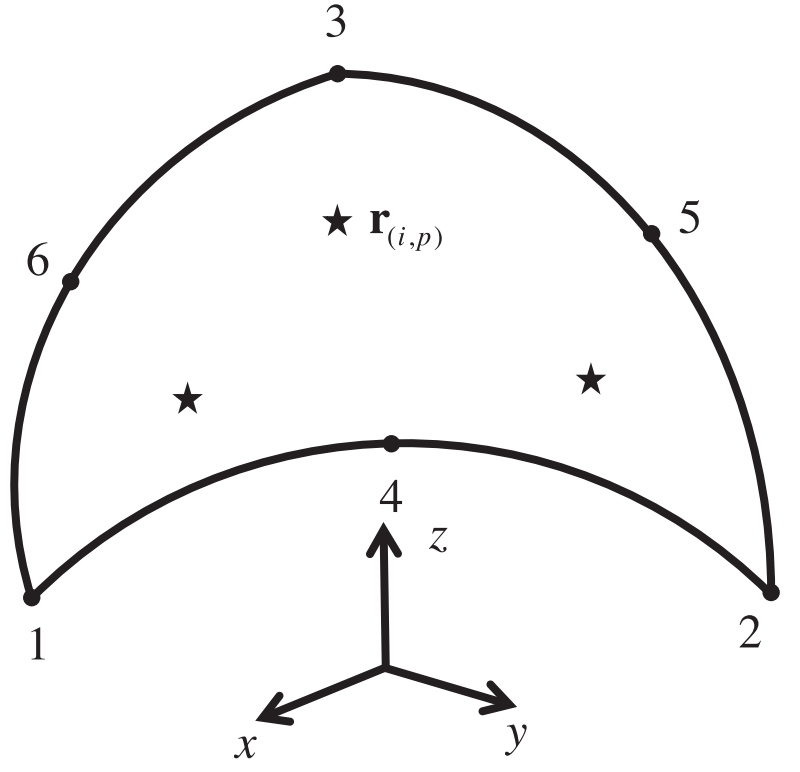

(a)

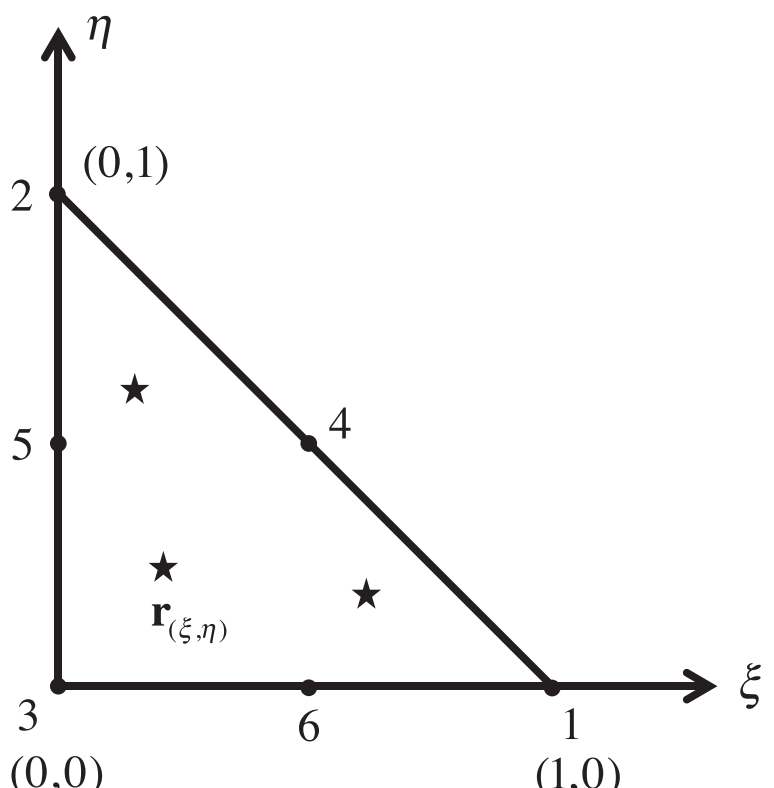

(b)

FIG. 1. (a) Curvilinear triangular patch in the Cartesian coordinate system. (b) Mapped right-angle triangular patch in the $(\xi, \eta)$ space.

mapped locations, which are denoted by $\mathbf{r}_{(\xi, \eta)}$, in the $(\xi, \eta)$ space, respectively.

To facilitate the reconstruction of $\varphi(\mathbf{r}, t)$ in Eq. (3), $\{\mathbf{I}(t)\}_{(i, p)}$ in Eq. (4) is temporally expanded/approximated using (shifted) Lagrange interpolation functions as ${ }^{25,34,35}$

$$
\{\mathbf{I}(t)\}_{(i, p)}=\sum_{l=1}^{N_{\mathrm{t}}}\left\{\mathbf{I}_{l}\right\}_{(i, p)} T(t-l \Delta t) .
$$

Here, $N_{\mathrm{t}}$ is the number of time steps, $\Delta t$ is the time step size, $T(t)$ is the Lagrange interpolation function, and $\left\{\mathbf{I}_{l}\right\}_{(i, p)}$ is the sample of $\{\mathbf{I}(t)\}_{(i, p)}$ at $t=l \Delta t$, i.e., $\left\{\mathbf{I}_{l}\right\}_{(i, p)}=\{\mathbf{I}(l \Delta t)\}_{(i, p)}$.

\section{Implicit MOT scheme}

Inserting Eqs. (4) and (5) into Eq. (3), point testing the resulting equation in space and time, i.e., evaluating it at spatial points $\mathbf{r}_{(j, q)}, j=1, \ldots, N_{\mathrm{u}}, q=1, \ldots, N_{\mathrm{p}}$, and at time samples $t=h \Delta t, h=1, \ldots, N_{\mathrm{t}}$, yield the implicit MOT system,

$$
\mathbf{Z}_{0}^{\mathrm{imp}} \mathbf{I}_{h}=\mathbf{V}_{h}^{\mathrm{i}}-\sum_{l=1}^{h-1} \mathbf{Z}_{h-l}^{\mathrm{imp}} \mathbf{I}_{l}, \quad h=1, \ldots, N_{\mathrm{t}}
$$

Here, the entries of the tested excitation vector $\mathbf{V}_{h}^{\mathrm{i}}$ and the implicit MOT matrices $\mathbf{Z}_{h-l}^{\text {imp }}$ are given by

$$
\begin{aligned}
\left\{\mathrm{V}_{h}^{\mathrm{i}}\right\}_{(j, q)}=\partial_{t} \varphi^{\mathrm{i}} & \left.\left(\mathrm{r}_{(j, q)}, t\right)\right|_{t=h \Delta t} \\
\left\{\mathbf{Z}_{h-l}^{\mathrm{imp}}\right\}_{(j, q)(i, p)}= & \left.\frac{1}{2} \vartheta^{-1}\left(\mathbf{r}_{(j, q)}\right) \partial_{t} T(t)\right|_{t=(h-l) \Delta t} \delta_{i j} \delta_{p q} \\
& -\int_{S_{p}} \vartheta^{-1}\left(\mathbf{r}^{\prime}\right) L_{(i, p)}\left(\mathbf{r}^{\prime}\right) \hat{\mathbf{n}}^{\prime}\left(\mathbf{r}^{\prime}\right) \cdot \frac{\mathbf{R}}{4 \pi R^{3}} \\
& \times\left[\partial_{t} T(t)+\frac{R}{c_{0}} \partial_{t}^{2} T(t)\right]_{t=(h-l) \Delta t-R / c_{0}} d s^{\prime}
\end{aligned}
$$

where $S_{p}$ is the surface of the $p$ th patch, $\mathbf{R}=\mathbf{r}_{(j, q)}$ $-\mathbf{r}^{\prime}, R=|\mathbf{R}|$, and $\delta_{i j}=1$ for $i=j$ and $\delta_{i j}=0$ for $i \neq j$. The method that is used for computing the entries of $\mathbf{Z}_{h-l}^{\text {imp }}$ is described in the Appendix.

After the matrices $\mathbf{Z}_{h-l}^{\text {imp }}$ (including $\mathbf{Z}_{0}^{\text {imp }}$ ) and the vector $\mathbf{V}_{h}^{\mathrm{i}}$ are computed and stored, unknown vectors $\mathbf{I}_{h}, h=1, \ldots, N_{\mathrm{t}}$, are obtained recursively by time marching, as briefly described next. First, $\mathbf{I}_{1}$ at $t=\Delta t$ is found by solving the MOT system in Eq. (6) with right-hand side $\mathbf{V}_{1}^{\mathrm{i}}$. Then at $t=2 \Delta t, \mathbf{I}_{1}$ is used to compute the matrix-vector product $\mathbf{Z}_{1}^{\text {imp }} \mathbf{I}_{1}$ [there is only one term in the summation on the right hand side of Eq. (6)], which is subtracted from $\mathbf{V}_{2}^{i}$ to yield the complete right-hand side. $\mathbf{I}_{2}$ is found by solving the MOT system in Eq. (6) with this right-hand side. Then, at $t=3 \Delta t, \mathbf{I}_{1}$ and $\mathbf{I}_{2}$ are used to compute the summation $\mathbf{Z}_{2}^{\mathrm{imp}} \mathbf{I}_{1}+\mathbf{Z}_{1}^{\mathrm{imp}} \mathbf{I}_{2}$, which is subtracted from $\mathbf{V}_{3}^{\mathrm{i}}$ to yield the complete right-hand side. This permits the computation of $\mathbf{I}_{3}$ and so on. At the end of time marching, all $\mathbf{I}_{h}, h=1, \ldots, N_{\mathrm{t}}$, are obtained known. Solution of the MOT system in Eq. (6), which is required by this time marching algorithm at every time step, is done using an iterative solver. In this work, a transpose-free quasi-minimal residual (TFQMR) scheme $^{36}$ is used for this purpose. Iterations of this scheme are terminated when $\left\|\mathbf{I}_{h}^{(n)}-\mathbf{I}_{h}^{(n-1)}\right\|<\chi^{\text {TFQMR }}$; here $\mathbf{I}_{h}^{(n)}$ and $\mathbf{I}_{h}^{(n-1)}$ are the solution vectors at two consecutive iterations ( $n$ and $n-1)$ and $\chi^{\mathrm{TFQMR}}$ is a user-defined tolerance/accuracy parameter.

\section{The explicit MOT scheme}

Inserting Eq. (4) into Eq. (3) and point testing the resulting equation in space, i.e., evaluating it at spatial points $\mathbf{r}_{(j, q)}, j$ $=1, \ldots, N_{\mathrm{u}}, q=1, \ldots, N_{\mathrm{p}}$, yield a linear system of ODEs,

$$
\mathbf{G} \partial_{t} \mathbf{I}(t)=\mathbf{V}^{\mathrm{i}}(t)+\mathbf{V}^{\mathrm{s}}(t) .
$$

Here, the entries of time dependent vectors $\mathbf{V}^{\mathrm{i}}(t)$ and $\mathbf{V}^{\mathrm{s}}(t)$, and the entries of the Gram matrix $\mathbf{G}$ are given by 


$$
\begin{aligned}
\{\mathbf{G}\}_{(j, q)(i, p)}= & \frac{1}{2} \vartheta^{-1}\left(\mathbf{r}_{(j, q)}\right) \delta_{i j} \delta_{p q}, \\
\left\{\mathbf{V}^{\mathrm{i}}(t)\right\}_{(j, q)}= & \partial_{t} \varphi^{\mathrm{i}}\left(\mathbf{r}_{(j, q)}, t\right), \\
\left\{\mathbf{V}^{\mathrm{s}}(t)\right\}_{(j, q)}= & \sum_{p=1}^{N_{\mathrm{p}}} \sum_{i=1}^{N_{\mathrm{u}}} \partial_{t} \int_{S_{p}} \vartheta^{-1}\left(\mathbf{r}^{\prime}\right) L_{(i, p)}\left(\mathbf{r}^{\prime}\right) \hat{\mathbf{n}}^{\prime}\left(\mathbf{r}^{\prime}\right) \\
& \cdot \nabla^{\prime} \frac{\left\{\mathbf{I}\left(t-R / c_{0}\right)\right\}_{(i, p)}}{4 \pi R} d s^{\prime} .
\end{aligned}
$$

Equation (9) is a system of ODEs and the samples of the unknown coefficient vector, i.e., $\mathbf{I}_{h}=\mathbf{I}(h \Delta t)$, is obtained by integrating this $\mathrm{ODE}$ system in time using a $\mathrm{PE}(\mathrm{CE})^{m}$-type linear multistep scheme. This approach calls for sampling Eq. (9) in time,

$$
\mathbf{G i}_{h}=\mathbf{V}_{h}^{\mathrm{i}}+\mathbf{V}_{h}^{\mathrm{s}},
$$

where $\dot{\mathbf{I}}_{h}=\left.\partial_{t} \mathbf{I}(t)\right|_{t=h \Delta t}, \mathbf{V}_{h}^{\mathrm{i}}=\mathbf{V}^{\mathrm{i}}(h \Delta t)$, and $\mathbf{V}_{h}^{\mathrm{s}}=\mathbf{V}^{\mathrm{s}}(h \Delta t)$. To compute $\mathbf{V}_{h}^{\mathrm{s}}$, one has to account for the retarded-time integral in Eq. (12); this is done by using temporal interpolation on $\left\{\mathbf{I}\left(t-R / c_{0}\right)\right\}_{(i, p)}$. Inserting Eq. (5) into Eq. (13) yields

$$
\mathbf{G} \dot{\mathbf{I}}_{h}=\mathbf{V}_{h}^{\mathrm{i}}-\sum_{l=1}^{h} \mathbf{Z}_{h-l}^{\exp } \mathbf{I}_{l}, \quad h=1, \ldots, N_{\mathrm{t}},
$$

where the entries of the explicit MOT matrices $\mathbf{Z}_{h-l}^{\exp }$ are given by

$$
\begin{aligned}
\left\{\mathbf{Z}_{h-l}^{\exp }\right\}_{(j, q)(i, p)}= & -\int_{S_{p}} \vartheta^{-1}\left(\mathbf{r}^{\prime}\right) L_{(i, p)}\left(\mathbf{r}^{\prime}\right) \hat{\mathbf{n}}^{\prime}\left(\mathbf{r}^{\prime}\right) \cdot \frac{\mathbf{R}}{4 \pi R^{3}} \\
& \times\left[\partial_{t} T(t)+\frac{R}{c_{0}} \partial_{t}^{2} T(t)\right]_{t=(h-l) \Delta t-R / c_{0}} d s^{\prime} .
\end{aligned}
$$

Equation (14) is a system of ODEs, which relates samples of the unknown's temporal derivative, $\dot{\mathbf{I}}_{h}$, to the samples of the unknown, $\mathbf{I}_{h}$. A $\mathrm{PE}(\mathrm{CE})^{m}$ method enhanced with an SOR scheme ${ }^{27,37}$ is used to for integrating Eq. (14) in time for the unknown samples $\mathbf{I}_{h}, h=1, \ldots, N_{\mathrm{t}}$. Steps of this method are described next.

Step 0: Before the time marching starts, compute and store $\mathbf{G}^{-1}$.

For $h=1, \ldots, N_{\mathrm{t}}$.

Step 1: Compute the fixed part (the part that does not change within the steps of one time step) of the right-hand side of Eq. (14),

$$
\mathbf{V}_{h}^{\exp }=\mathbf{V}_{h}^{\mathrm{i}}-\sum_{l=1}^{h-1} \mathbf{Z}_{h-l}^{\exp } \mathbf{I}_{l} .
$$

Step 2: Predict $\mathbf{I}_{h}$ using $K$ past values of $\mathbf{I}_{l}$ and $\dot{\mathbf{I}}_{l}$,

$$
\mathbf{I}_{h}=\sum_{k=1}^{K}\left[\{\mathbf{p}\}_{k} \mathbf{I}_{h-1+k-K}+\{\mathbf{p}\}_{K+k} \dot{\mathbf{I}}_{h-} 1+k-K\right] .
$$
of $\mathbf{I}_{h}$,

Step 3: Evaluate $\dot{\mathbf{I}}_{h}$ using $\mathbf{V}_{h} \exp$ and the predicted value

$$
\dot{\mathbf{I}}_{h}=\mathbf{G}^{-1}\left(\mathbf{V}_{h}^{\exp }-\mathbf{Z}_{0}^{\exp } \mathbf{I}_{h}\right) .
$$

Step 4: Set $\dot{\mathbf{I}}_{h}^{(0)}=\dot{\mathbf{I}}_{h}$ and start "correcting" $\mathbf{I}_{h}^{(n)}$ and $\dot{\mathbf{I}}_{h}^{(n)}, n=1, \ldots, m$, recursively, until convergence.

For $n=1, \ldots, m$. and $\dot{\mathbf{I}}_{l}$,

Step 4.1: Correct $\mathbf{I}_{h}^{(n)}$ using $\dot{\mathbf{I}}_{h}^{(n-1)}$ and $K$ past values of $\mathbf{I}_{l}$

$$
\begin{aligned}
\mathbf{I}_{h}^{(n)}= & \sum_{k=1}^{K}\left[\{\mathbf{c}\}_{k} \mathbf{I}_{h-1+k-K}+\{\mathbf{c}\}_{K+k} \dot{\mathbf{I}}_{h-1+k-K}\right] \\
& +\{\mathbf{c}\}_{2 k+1} \dot{\mathbf{I}}_{h}^{(n-1)} .
\end{aligned}
$$

Step 4.2: Update $\mathbf{I}_{h}^{(n)}$ using $\mathbf{I}_{h}^{(n)}$ and $\mathbf{I}_{h}^{(n-1)}$,

$$
\mathbf{I}_{h}^{(n)}=\alpha \mathbf{I}_{h}^{(n)}+(1-\alpha) \mathbf{I}_{h}^{(n-1)} .
$$

Step 4.3: Evaluate $\dot{\mathbf{I}}_{h}^{(n)}$ as using $\mathbf{V}_{h}^{\exp }$ and the corrected value of $\mathbf{I}_{h}$,

$$
\dot{\mathbf{I}}_{h}^{(n)}=\mathbf{G}^{-1}\left(\mathbf{V}_{h}^{\exp }-\mathbf{Z}_{0}^{\exp } \mathbf{I}_{h}^{(n)}\right) .
$$

Step 4.4: Check convergence,

$$
\left\|\mathbf{I}_{h}^{(n)}-\mathbf{I}_{h}^{(n-1)}\right\|<\chi^{\mathrm{PECE}} .
$$

End for loop over $n$.

Step 5: Assuming convergence happens at the $m$ th iteration, set $\mathbf{I}_{h}=\mathbf{I}_{h}^{(m)}$ and $\dot{\mathbf{I}}_{h}=\dot{\mathbf{I}}_{h}^{(m)}$.

End for loop over $h$.

Several comments about the explicit MOT scheme described above are in order.

(i) At Step 4.4, $\chi^{\mathrm{PECE}}$ is a user-defined tolerance/accuracy parameter.

(ii) At Steps 2 and 4.1, $\mathbf{p}$ and $\mathbf{c}$ are vectors of length $2 K$ and $2 K+1$, which store the predictor and corrector coefficients of the $\mathrm{PE}(\mathrm{CE})^{m}$ scheme. These coefficients can be obtained by either applying polynomial interpolation between time samples (e.g., AdamMoulton, Adam-Bashforth, and backward difference methods ${ }^{38}$ ) or using a numerical scheme that approximates the solution in terms of decaying and oscillating exponentials. ${ }^{39}$

(iii) Initial values of $\mathbf{I}_{l}$ and $\dot{\mathbf{I}}_{l}$ are assumed to be zero, i.e., $\mathbf{I}_{l}=0$ and $\dot{\mathbf{I}}_{l}=0$, for $l=0, \ldots, K-1$. This does not introduce any significant error in the solution since $\varphi^{\mathrm{i}}(\mathbf{r}, t)$ and $p^{\mathrm{i}}(\mathbf{r}, t)$ are vanishingly small for $t \leq 0, \forall \mathbf{r} \in S$. If another type of excitation, which increases faster at the beginning of time marching, is used, the initialization of $\mathbf{I}_{l}$ and $\dot{\mathbf{I}}_{l}$ can be done using the Euler method or spectral-deferred correction type methods. ${ }^{38,40}$

(iv) Comparing the expressions of the entries of $\mathbf{Z}_{h-l}^{\exp }$ in Eq. (15) to those of $\mathbf{Z}_{h-l}^{\mathrm{imp}}$ in Eq. (8), it can be seen that $\mathbf{Z}_{h-l}^{\exp }$ and $\mathbf{Z}_{h-l}^{\text {imp }}$ are the same except the "self-term" expression $\left.\vartheta^{-1}\left(\mathbf{r}_{(j, q)}\right) \partial_{t} T(t)\right|_{t=(h-l) \Delta t} \delta_{i j} \delta_{p q} / 2$ presented in $\mathbf{Z}_{h-l}^{\text {imp }}$. This term contributes only to the diagonal entries of $\mathbf{Z}_{h-l}^{\mathrm{imp}}, h-l=0,1, \ldots, P$, where $P$ is the 
order of $T(t)$, since $\left.\partial_{t} T(t)\right|_{t=(h-l) \Delta t}=0$ for $h-l \notin$ $\{0,1, \ldots, P\}$ and $\delta_{i j}=0$ for $i \neq j$ and $\delta_{p q}=0$ for $p \neq q$. Since the same diagonal entries of $\mathbf{Z}_{h-l}^{\exp }$ are already non-zero, the sparseness levels of $\mathbf{Z}_{h-l}^{\text {exp }}$ and $\mathbf{Z}_{h-l}^{\mathrm{imp}}$ are the same for all the values of $l, l=1, \ldots, h$. The effect of this on the computational complexity of the implicit and explicit MOT schemes is discussed in Sec. IIE. The method, which is used for computing the entries of $\mathbf{Z}_{h-l}^{\mathrm{imp}}$ and described in the Appendix, is also used for computing entries of $\mathbf{Z}_{h-l}^{\exp }$.

\section{E. Computational complexity analysis}

Numerical results presented in Sec. III show that the explicit MOT scheme maintains its stability using a $\Delta t$ as large as that would be used by the implicit scheme. The results also show that for the same $\Delta t$, both schemes achieve the same level of accuracy. Therefore, while comparing the computational complexities of these two schemes, it is assumed that they use the same $\Delta t$ and they are executed for the same $N_{\mathrm{t}}$. To this end, in this section, the computational costs of one time step for both schemes are compared.

At a given time step, the implicit MOT scheme computes $\mathbf{V}_{h}^{\mathrm{imp}}=\mathbf{V}_{h}^{\mathrm{i}}-\boldsymbol{\Sigma}_{l=1}^{h-1} \mathbf{Z}_{h-l}^{\mathrm{imp}} \mathbf{I}_{l}$ [the right hand side of Eq. (6)] while the explicit MOT scheme computes $\mathbf{V}_{h}^{\exp }$ $=\mathbf{V}_{h}^{\mathrm{i}}-\Sigma_{l=1}^{h-1} \mathbf{Z}_{h-l}^{\text {exp }} \mathbf{I}_{l}$ [Eq. (16) and the fixed part of the right hand side of Eq. (14)]. The sparseness levels of $\mathbf{Z}_{h-l}^{\exp }$ and $\mathbf{Z}_{h-l}^{\text {imp }}$ are the same for $l=1, \ldots, h$ as discussed in Sec. II D. This means that the costs of computing the summations in $\mathbf{V}_{h}^{\mathrm{imp}}$ and $\mathbf{V}_{h}^{\text {exp }}$ are the same. It should also be noted here that this computation could be accelerated using the PWTD algorithm ${ }^{11,12,21,22}$ or the FFT-based methods, ${ }^{23-26}$ both the explicit and implicit MOT scheme benefit from the use of these methods. Therefore, with or without an acceleration method, the cost of computing $\mathbf{V}_{h}^{\text {imp }}$ and $\mathbf{V}_{h}^{\exp }$ is the same and it is represented by $C^{\text {rhs }}$.

At every time step, the implicit MOT scheme iteratively solves the matrix system in Eq. (6). The computational complexity of this operation scales as

$$
C^{\mathrm{imp}} \sim O\left(N_{\mathrm{iter}}^{\mathrm{imp}} F_{\text {iter }}^{\mathrm{imp}} \gamma N_{\mathrm{s}}\right)
$$

Here, $N_{\mathrm{s}}=N_{\mathrm{p}} N_{\mathrm{u}}$ represents the total number of unknowns, $N_{\text {iter }}^{\mathrm{imp}}$ is the number of iterations, $F_{\text {iter }}^{\mathrm{imp}}$ is the number of matrix-vector multiplications at each iteration, and $\gamma$ is the "denseness" factor of $\mathbf{Z}_{0}^{\text {imp }}$, i.e., (average) number of nonzero entries in one row/column of $\mathbf{Z}_{0}^{\text {imp }}$.

The computational cost of the explicit MOT scheme is derived by counting the number of operations in steps $1-4$ of the $\mathrm{PE}(\mathrm{CE})^{m}$ scheme. The cost at step 1, i.e., the cost of computing $\mathbf{V}_{h}^{\exp }$, is already discussed above. The computational cost of the remaining steps $2-4$ is given by

$$
\begin{aligned}
C^{\exp \sim} & O\left(2 K N_{\mathrm{s}}\right)+O\left(m[2 K+1] N_{\mathrm{s}}\right) \\
& +O\left([m+1] \gamma N_{\mathrm{s}}\right)+O\left([m+1] N_{\mathrm{s}}\right) .
\end{aligned}
$$

Here, the first term is the cost of computing the right hand side of Eq. (17), the second term is the cost of computing the right hand side of Eq. (19) for a total of $m$ iterations. The third term is the total cost of multiplications $\mathbf{Z}_{0}^{\exp } \mathbf{I}_{h}$ in Eq. (18) and $\mathbf{Z}_{0}^{\exp } \mathbf{I}_{h}^{(n)}$ ( $m$ times) in Eq. (21). Note that $\gamma$ is the denseness factor of $\mathbf{Z}_{0}^{\text {exp }}$ (same as that of $\mathbf{Z}_{0}^{\text {imp }}$ ). The last term is total cost of multiplication with $\mathbf{G}^{-1}$ in Eqs. (18) and (21).

To compare $C^{\text {imp }}$ and $C^{\exp }$, the values of $m, K, \gamma$, $N_{\mathrm{s}}, N_{\text {iter }}^{\mathrm{imp}}$, and $F_{\text {iter }}^{\mathrm{imp}}$ are needed to be studied. In the case of high frequency excitations (small $\Delta t, \Delta t \ll D_{\max } / c_{0}$, where $D_{\max }$ is the maximum distance between any two points in $S$ ), both $\mathbf{Z}_{0}^{\text {imp }}$ and $\mathbf{Z}_{0}^{\text {exp }}$ are very sparse, i.e., $\gamma \ll N_{\text {s }}$. Therefore, $C^{\text {imp }}$ and $C^{\exp }$ scale as $C^{\text {imp }} \sim O\left(N_{\text {iter }}^{\text {imp }} F_{\text {iter }}^{\text {imp }} \gamma N_{\mathrm{s}}\right)$ and $C^{\exp }$ $\sim O\left(m K N_{\mathrm{s}}\right)+O\left(m \gamma N_{\mathrm{s}}\right)$. This suggests that $C^{\exp } \approx C^{\mathrm{imp}}$ (depending on the exact values of $m, K, \gamma, N_{\text {iter }}^{\text {imp }}$, and $F_{\text {iter }}^{\text {imp }}$ ), which means that both solvers will have similar speeds for high frequency excitation ( ince $C^{\exp }+C^{\text {rhs }} \approx C^{\text {imp }}+C^{\text {rhs }}$ ). This is indeed demonstrated by the numerical results presented in Sec. III A.

Matrices $\mathbf{Z}_{h-l}^{\text {imp }}=0$ and $\mathbf{Z}_{h-l}^{\exp }=0$ for $h-l>D_{\max } /$ $\left(c_{0} \Delta t\right)+P$. Consequently, as the frequency decreases (large $\left.\Delta t, \Delta t \approx D_{\max } / c_{0}\right)$, the number of non-zero $\mathbf{Z}_{h-l}^{\text {imp }}$ and $\mathbf{Z}_{h-l}^{\exp }$ decreases. However, these non-zero matrices (including $\mathbf{Z}_{0}^{\text {imp }}$ and $\mathbf{Z}_{0}^{\exp }$ ) become denser (even full matrices). For example, for $D_{\max } /\left(c_{0} \Delta t\right)<1$, all non-zero $\mathbf{Z}_{h-l}^{\text {imp }}$ and $\mathbf{Z}_{h-l}^{\exp }$ are completely full. This means that $C^{\text {rhs }}$ (cost of computing $\mathbf{V}_{h}^{\text {imp }} / \mathbf{V}_{h}^{\text {exp }}$ ) becomes significantly smaller than $C^{\text {imp }}$ and $C^{\exp }$ and the latter can be used to predict the total cost of time marching. As $\mathbf{Z}_{0}^{\text {imp }}$ and $\mathbf{Z}_{0}^{\text {exp }}$ become denser, $\gamma \sim N_{\mathrm{s}}$. As a result, $C^{\text {imp }} \sim O\left(N_{\text {iter }}^{\text {imp }} F_{\text {iter }}^{\text {imp }} N_{\mathrm{s}}^{2}\right)$ and $C^{\exp } \sim O\left(m N_{\mathrm{s}}^{2}\right)$. Since $m$ is smaller than $N_{\text {iter }}^{\text {imp }} F_{\text {iter }}^{\text {imp }}$ (as shown by the numerical results presented in Sec. III A), $C^{\mathrm{imp}}<C^{\exp }$. As a result, for large $\Delta t$, the explicit MOT solver is expected to be faster than its implicit counterpart. Indeed, this is demonstrated by the numerical results presented in Sec. III A.

\section{F. Comments}

Several comments about the surface integral equation formulation described in Sec. II A and its discretization using the methods described in Sec. II B, IIC, and IID are in order.

(1) The TDKIE in Eq. (3) is formulated to analyze scattering from an acoustically rigid object. A perfectly rigid scatterer can be considered as a mathematical idealization since it refers to an object made of an infinitely stiff material. However, it can be considered as a good approximation in scattering scenarios where an object with a high bulk modulus resides in a background medium with a significantly lower bulk modulus. For example, a steel submarine embedded in water can be approximated as an acoustically rigid scatterer since the bulk modulus of steel is roughly 70 times that of water. For scenarios that do not fall in this category, where the object has to be considered as a penetrable scatterer, one can formulate a coupled system of surface integral equations by combining exterior and interior scattering integrals. ${ }^{30}$

(2) The TDKIE in Eq. (3) suffers from the well-known internal resonance problem. ${ }^{41}$ However, the amplitude of spurious modes can be kept under control (for only 
TDSIEs but not their frequency domain versions) by increasing the accuracy of the MOT scheme. ${ }^{42,43}$ These modes can be completely eliminated from the solution if one solves a combination of the TDKIE in Eq. (3) and its normal derivative (Burton-Miller formulation). ${ }^{10}$ However, this formulation calls for significantly more complicated singularity extraction/cancellation techniques (especially for higher-order surface discretizations like the one described in Sec. II B) since the order of singularity in the kernel of the integral equation is increased. ${ }^{44}$

(3) The MOT schemes developed for solving the TDKIE in Eq. (3) can be extended to solve the coupled system of equations to account for penetrable scatterers or the Burton-Miller surface integral equation to obtain a more accurate solution. These extensions do not call for significant changes in either the method used for the temporal discretization or the implementation of the time marching. However, one needs to significantly modify the method used for the spatial discretization to account for different integral equation kernels. These modifications will be formulated and described in a future publication.

\section{NUMERICAL RESULTS}

In this section, the accuracy, efficiency, and applicability of the proposed explicit MOT are demonstrated through the numerical analysis of transient acoustic scattering from rigid objects residing in an unbounded background medium. In all examples, the object/scatterer is excited by a plane wave with velocity potential

$$
\varphi^{\mathrm{i}}(\mathbf{r}, t)=\varphi_{0}^{\mathrm{i}} G_{\bmod }\left(t-\hat{\mathbf{k}}^{\mathrm{i}} \cdot \mathbf{r} / c_{0}\right) .
$$

Here, $\varphi_{0}^{\mathrm{i}}=1 \mathrm{~m}^{2} / \mathrm{s}$ is the amplitude, $\hat{\mathbf{k}}^{\mathrm{i}}$ is the direction of propagation of the incident plane wave, $G \bmod (t)$ $=\cos \left[2 \pi f_{0}\left(t-t_{0}\right)\right] e^{-\left(t-t_{0}\right)^{2} / 2 w^{2}}$ is a modulated Gaussian pulse, $f_{0}$ is the pulse center frequency, $t_{0}$ is the pulse delay, and $w$ is a measure of the pulse width. For all the examples considered here, an effective bandwidth for $G_{\bmod }(t)$ is defined as $f_{\mathrm{bw}}=3 /(2 \pi w)$. This specific definition $f_{\mathrm{bw}}$ ensures that $0.003 \%$ of $G_{\text {mod }}(t)$ 's total power is within the frequency band $\left[f_{0}-f_{\mathrm{bw}}, f_{0}+f_{\mathrm{bw}}\right]$. In all examples, it is assumed that the scatterer resides under water and therefore $c_{0}=1500 \mathrm{~m} / \mathrm{s}$. The spatial discretization uses the Nyström method with second-order Lagrange polynomials, i.e., $N_{\mathrm{u}}=6$. Tolerance/ accuracy parameters for the implicit and explicit MOT schemes are the same: $\chi^{\mathrm{PECE}}=\chi^{\mathrm{TFQMR}}=1 \times 10^{-13}$.

After the time-domain simulations are completed and the unknown coefficients $\mathbf{I}_{h}=\mathbf{I}(h \Delta t), h=1, \ldots, N_{\mathrm{t}}$ are solved for and stored, one can obtain the time-harmonic total velocity potential $\varphi(\mathbf{r}, f)$ at frequency $f$ using

$$
\varphi(\mathbf{r}, f)=\sum_{p=1}^{N_{\mathrm{p}}} \sum_{i=1}^{N_{\mathrm{u}}}\{\mathbf{I}(f)\}_{(i, p)} \vartheta^{-1}(\mathbf{r}) L_{(i, p)}(\mathbf{r}) .
$$

Here, $\mathbf{I}(f)$ is the vector of time-harmonic expansion coefficients and its entries are obtained by dividing the Fourier transform of $\mathbf{I}(t)$ 's corresponding entries by the Fourier transform of $G_{\text {mod }}(t)$. Then, $\varphi(\mathbf{r}, f)$ is used to compute the scattering cross section (SCS) of the object $\sigma(\theta, \phi, f)$ defined as ${ }^{45}$

$$
\begin{aligned}
\sigma(\theta, \phi, f)= & \lim _{\mathbf{r} \rightarrow \infty} 4 \pi r^{2}\left|\varphi^{\mathrm{s}}(\mathbf{r}, f)\right|^{2} /\left|\varphi_{0}^{\mathrm{i}}\right|^{2} \\
= & \left|\int_{S} \varphi\left(\mathbf{r}^{\prime}, f\right) \hat{\mathbf{n}}^{\prime}\left(\mathbf{r}^{\prime}\right) \cdot k_{0} \hat{\mathbf{k}}^{\mathrm{s}} e^{j k_{0} \hat{\mathbf{k}}^{\mathrm{s}} \cdot \mathbf{r}^{\prime}} d s^{\prime}\right|^{2} / \\
& \left(4 \pi\left|\varphi_{0}^{\mathrm{i}}\right|^{2}\right) .
\end{aligned}
$$

Here, $\varphi^{\mathrm{s}}(\mathbf{r}, f)$ is the time-harmonic scattered velocity potential at frequency $f, k_{0}=2 \pi f / c_{0}$ is the wavenumber in the background medium, and $\hat{\mathbf{k}}^{\mathrm{s}}=\hat{\mathbf{x}} \sin \theta \cos \phi+\hat{\mathbf{y}} \sin \theta \sin \phi$ $+\hat{\mathbf{z}} \cos \phi$ is the direction along which $\sigma(\theta, \phi, f)$ is computed. Note that the traditional definitions of the angles $\theta$ and $\phi$ in spherical coordinate system are used here: $\theta$ is measured from the $z$-axis and $\phi$ is measured on the $x y$-plane from the $x$ axis.

\section{A. Accuracy and efficiency}

For this example, transient acoustic scattering from a rigid unit sphere is analyzed using the implicit and explicit MOT schemes. For the first set of simulations, the sphere surface is discretized into $N_{\mathrm{p}}=2560$ curvilinear triangular patches resulting in $N_{\mathrm{s}}=15360$ number of unknowns. The temporal interpolation function $T(t)$ is the fourth-order Lagrange polynomial, i.e., $P=4$. For the explicit MOT solver, the SOR coefficient $\alpha$ $=1$ (i.e., no SOR is applied) and the coefficients $\mathbf{p}$ and $\mathbf{c}$ are computed using the numerical scheme that approximates the solution in terms of decaying and oscillating exponentials, ${ }^{39}$ resulting in a $\mathrm{PE}(\mathrm{CE})^{m}$ scheme with $K=22$. Excitation parameters $\hat{\mathbf{k}}^{\mathrm{i}}=\hat{\mathbf{z}}, f_{0}=874.64 \mathrm{~Hz}, f_{\mathrm{bw}}=273.3 \mathrm{~Hz}, w=1.747 \mathrm{~ms}$, and $t_{0}=13.98 \mathrm{~ms}$; and the simulations are carried out for $N_{\mathrm{t}}=2000$ time steps with $\Delta t=0.029 \mathrm{~ms}$. Figure 2 compares $\left|\{\mathbf{I}(t)\}_{(i, p)}\right|, p=3, i=3$, computed by the implicit and explicit MOT solvers at $\mathbf{r}_{(i, p)}=(-0.239,0.943,-0.233) \mathrm{m}$. The figure clearly shows that the results agree very well.

Let the SCS values, which are obtained using Eq. (27) with $f=f_{0}, \theta=\left[0^{\circ}, 180^{\circ}\right]$, and $\phi=0^{\circ}$ from the solutions computed by the explicit and implicit MOT solvers, be represented by $\sigma^{\exp }\left(\theta, 0^{\circ}, f_{0}\right)$ and $\sigma^{\operatorname{imp}}\left(\theta, 0^{\circ}, f_{0}\right)$, respectively. The

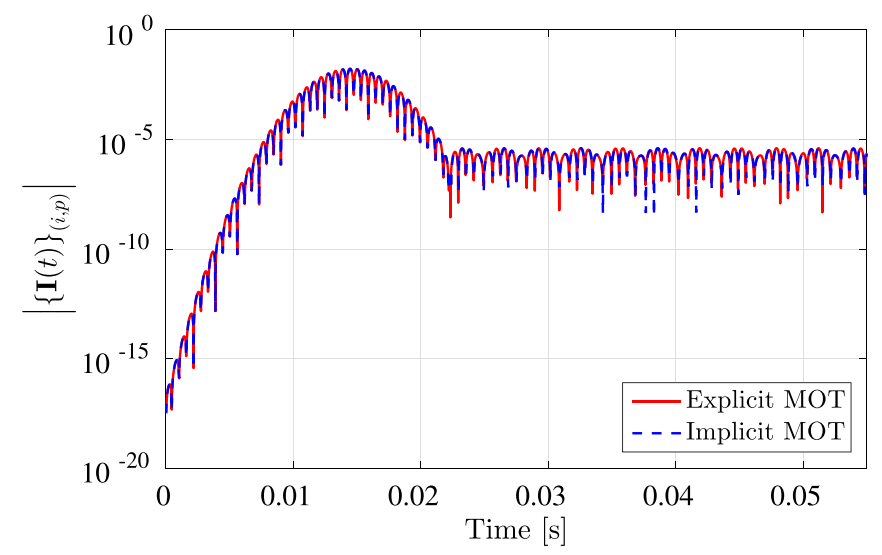

FIG. 2. (Color online) Comparison of $\left|\{\mathbf{I}(t)\}_{(i, p)}\right|, p=3, i=3$, computed by the implicit and explicit MOT solvers at $\mathbf{r}_{(i, p)} \stackrel{(i, p)}{=}(-0.239,0.943,-0.233) \mathrm{m}$. 


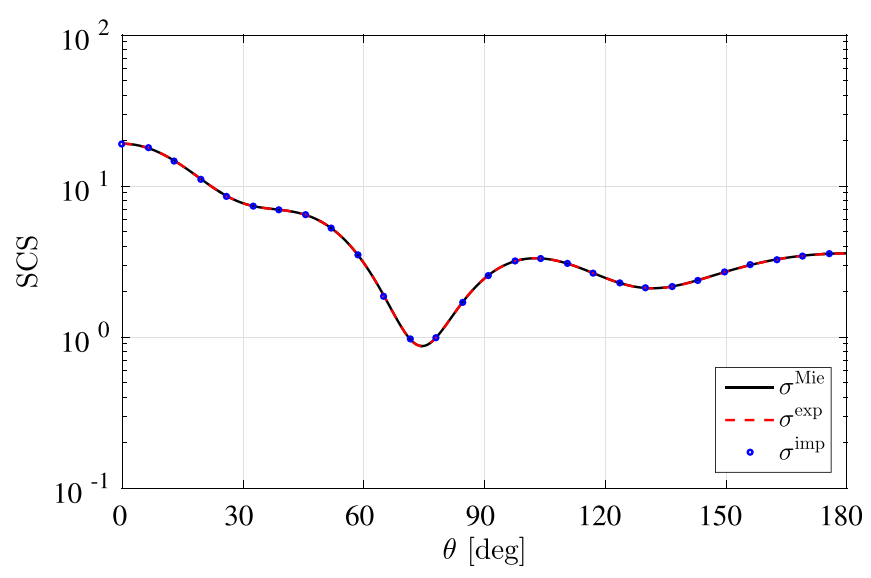

(a)

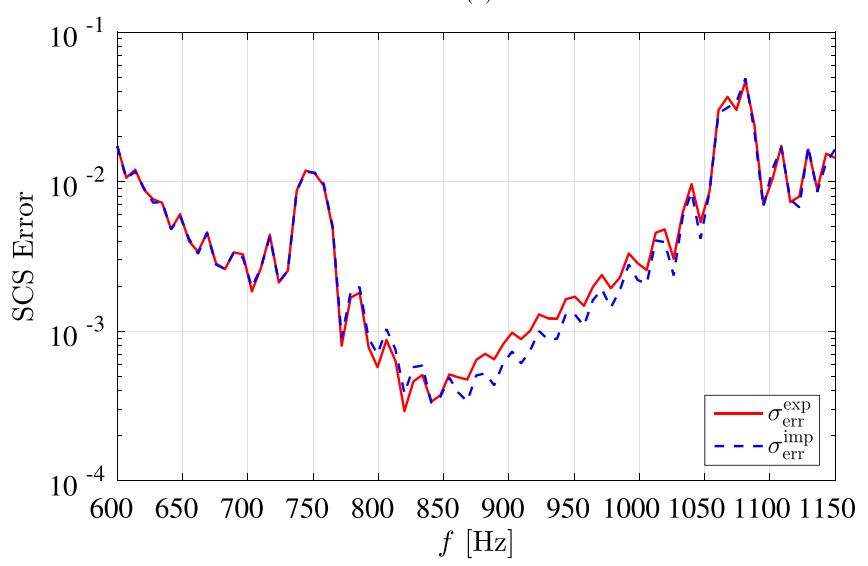

(b)

FIG. 3. (Color online) (a) Comparison of the SCS values obtained using Eq. (27) for $f=f_{0}, \theta=\left[0^{\circ}, 180^{\circ}\right]$, and $\phi=0^{\circ}$ from the solutions computed by the implicit and explicit MOT solvers to the SCS obtained from the Mie series solution. (b) Comparison of the relative error in SCS obtained using Eq. (27) for $f, \theta=\left[0^{\circ}, 180^{\circ}\right]$, and $\phi=0^{\circ}$ from the solutions computed by the implicit and explicit MOT solvers.

SCS of the sphere is also obtained at the same frequency and angles from the (analytical) Mie series solution. ${ }^{46}$ Let this value be represented by $\sigma^{\operatorname{Mie}}\left(\theta, 0^{\circ}, f_{0}\right)$. Figure 3(a) plots $\sigma^{\mathrm{Mie}}\left(\theta, 0^{\circ}, f_{0}\right), \sigma^{\mathrm{imp}}\left(\theta, 0^{\circ}, f_{0}\right)$, and $\sigma^{\exp }\left(\theta, 0^{\circ}, f_{0}\right)$ versus $\theta$. It shows that both solvers produce accurate results. To compare the accuracy of the implicit and explicit MOT solvers in the above simulations, the $L_{2}$-norm error in SCS is defined as

$$
\sigma_{\mathrm{err}}^{\mathrm{type}}(f)=\sqrt{\frac{\sum_{n=0}^{360}\left|\sigma^{\mathrm{Mie}}(n \Delta \theta, \phi, f)-\sigma^{\mathrm{type}}(n \Delta \theta, \phi, f)\right|^{2}}{\sum_{n=0}^{360}\left|\sigma^{\mathrm{Mie}}(n \Delta \theta, \phi, f)\right|^{2}}},
$$

where type $\in\{\operatorname{imp}, \exp \}, \Delta \theta=0.5^{\circ}$, and $\phi=0^{\circ}$. Figure 3(b) plots $\sigma_{\mathrm{err}}^{\operatorname{imp}}(f)$ and $\sigma_{\operatorname{err}}^{\exp }(f)$ versus $f$ changing from 600 to $1150 \mathrm{~Hz}$. It is clearly seen that both solvers provide accurate results within the effective band of the excitation.

For the next set of simulations, the sphere surface is discretized into $N_{\mathrm{p}}=116, N_{\mathrm{p}}=396$, and $N_{\mathrm{p}}=1126$ curvilinear triangular patches, resulting in $N_{\mathrm{s}}=696, N_{\mathrm{s}}=2376$, and $N_{\mathrm{s}}$ $=6756$ numbers of unknowns, respectively. The temporal interpolation function $T(t)$ is the third-order Lagrange polynomial, i.e., $P=3$. For the explicit MOT solver, the SOR coefficient is $\alpha=0.6$ and the coefficients $\mathbf{p}$ and $\mathbf{c}$ are computed using the numerical scheme that approximates the solution in terms of decaying and oscillating exponentials, ${ }^{39}$ resulting in a $\mathrm{PE}(\mathrm{CE})^{m}$ scheme with $K=22$. For each mesh, four different simulations with different $f_{0}$ and $w$ are executed using the explicit and implicit MOT solvers (a total of 24 solver simulations). For these sets of simulations, $\hat{\mathbf{k}}^{\mathrm{i}}=\hat{\mathbf{z}}, f_{0}=\{20,65$, $220,350\} \mathrm{Hz}, f_{\mathrm{bw}}=0.3125 f_{0}, w=3 /\left(0.625 \pi f_{0}\right)$, and $t_{0}=6 w$; and the simulations are carried out for $N_{\mathrm{t}}=600$ time steps with $\Delta t=1 /\left(32.5 f_{0}\right)$. Different $\Delta t$ results in a different value for the denseness of $\mathbf{Z}_{0}^{\mathrm{imp}}$ and $\mathbf{Z}_{0}^{\exp }: \gamma=\{696,106,30,22\}$, $\gamma=\{2376,309,58,34\}$, and $\gamma=\{6756,857,130,61\}$ for $N_{\mathrm{s}}$ $=696, N_{\mathrm{s}}=2376$, and $N_{\mathrm{s}}=6756$, respectively.

Table I compares the times required by different stages of the implicit and explicit MOT schemes summed over all $N_{\mathrm{t}}$ time steps: $T_{\text {fix }}^{\mathrm{imp}}$ and $T_{\text {fix }}^{\text {exp }}$ are the times required to compute $\mathbf{V}_{h}^{\mathrm{imp}}$ and $\mathbf{V}_{h}^{\mathrm{exp}}$, respectively, $T_{\mathrm{TFQMR}}^{\mathrm{imp}}$ is the time required by the TFQMR to solve the matrix system in Eq. (6), $T_{\mathrm{PECE}}^{\exp }$

TABLE I. Comparison of CPU times required by different stages of the implicit and explicit MOT schemes summed over all $N_{\mathrm{t}}$ time steps.

\begin{tabular}{|c|c|c|c|c|c|c|c|c|c|c|c|}
\hline \multirow[b]{2}{*}{$N_{\mathrm{s}}$} & \multirow[b]{2}{*}{$f_{0}[\mathrm{~Hz}]$} & \multicolumn{4}{|c|}{ Implicit MOT } & \multicolumn{6}{|c|}{ Explicit MOT } \\
\hline & & $T_{\text {fix }}^{\mathrm{imp}}[\mathrm{s}]$ & $T_{\mathrm{TFQMR}}^{\mathrm{imp}}[\mathrm{s}]$ & $T_{\text {tot }}^{\mathrm{imp}}[\mathrm{s}]$ & $\left\{N_{\text {iter }}^{\text {imp }} F_{\text {iter }}^{\text {imp }}\right\}_{\text {avg }}$ & $T_{\text {fix }}^{\exp }[\mathrm{s}]$ & $T_{\text {PECE }}^{\exp }[\mathrm{s}]$ & $T_{\text {tot }}^{\exp }[\mathrm{s}]$ & $m_{\text {avg }}$ & $\frac{T_{\mathrm{TFQMR}}^{\mathrm{imp}}}{T_{\mathrm{PECE}}^{\exp }}$ & $\frac{T_{\text {tot }}^{\mathrm{imp}}}{T_{\text {tot }}^{\exp }}$ \\
\hline 696 & 20 & 13.31 & 114.13 & 127.47 & 46.86 & 13.20 & 35.37 & 48.58 & 13.5 & 3.23 & 2.62 \\
\hline 696 & 65 & 16.73 & 18.37 & 35.11 & 48.77 & 17.17 & 6.63 & 23.82 & 15.09 & 2.77 & 1.47 \\
\hline 696 & 220 & 18.88 & 5.95 & 24.85 & 50.77 & 18.66 & 3.10 & 21.78 & 20.77 & 1.92 & 1.14 \\
\hline 696 & 350 & 18.48 & 4.37 & 22.87 & 50.93 & 18.56 & 2.37 & 20.95 & 20.20 & 1.84 & 1.09 \\
\hline 2376 & 20 & 153.35 & 967.69 & 1121.17 & 34.04 & 154.09 & 372.99 & 527.19 & 12.09 & 2.59 & 2.13 \\
\hline 2376 & 65 & 192.94 & 130.70 & 323.70 & 35.20 & 193.54 & 56.83 & 250.46 & 13.95 & 2.30 & 1.29 \\
\hline 2376 & 220 & 203.30 & 26.39 & 229.72 & 36.79 & 205.44 & 17.50 & 223.02 & 20.14 & 1.51 & 1.03 \\
\hline 2376 & 350 & 206.92 & 16.12 & 223.09 & 37.10 & 207.33 & 11.22 & 218.63 & 19.61 & 1.44 & 1.02 \\
\hline 6756 & 20 & 1237.76 & 6980.40 & 8219.66 & 30.41 & 1245.25 & 2816.47 & 4062.05 & 11.36 & 2.48 & 2.02 \\
\hline 6756 & 65 & 1534.39 & 917.32 & 2452.00 & 31.31 & 1535.08 & 418.07 & 1953.44 & 13.09 & 2.19 & 1.26 \\
\hline 6756 & 220 & 1595.66 & 146.16 & 1742.00 & 32.63 & 1641.22 & 99.85 & 1741.33 & 19.57 & 1.46 & 1.00 \\
\hline 6756 & 350 & 1648.97 & 72.84 & 1721.99 & 32.98 & 1626.80 & 50.68 & 1677.75 & 18.98 & 1.44 & 1.03 \\
\hline
\end{tabular}


TABLE II. Comparison of relative error in the SCS values obtained using Eq. (27) for $f=f_{0}, \theta=\left[0^{\circ}, 180^{\circ}\right]$ and $\phi=0^{\circ}$ from the solutions computed by the implicit and explicit MOT solvers.

\begin{tabular}{lrcc}
\hline \hline$N_{\mathrm{s}}$ & $f_{0}[\mathrm{~Hz}]$ & $\sigma_{\text {err }}^{\text {imp }}\left(f_{0}\right)$ & $\sigma_{\text {err }}^{\exp }\left(f_{0}\right)$ \\
\hline 696 & 20 & 0.0040 & 0.0035 \\
696 & 65 & 0.0044 & 0.0039 \\
696 & 220 & 0.0070 & 0.0069 \\
696 & 350 & 0.0123 & 0.0123 \\
2376 & 20 & 0.0007 & 0.0003 \\
2376 & 65 & 0.0012 & 0.0008 \\
2376 & 220 & 0.0045 & 0.0043 \\
2376 & 350 & 0.0109 & 0.0109 \\
6756 & 20 & 0.0006 & 0.0002 \\
6756 & 65 & 0.0011 & 0.0007 \\
6756 & 220 & 0.0044 & 0.0043 \\
6756 & 350 & 0.0109 & 0.0109 \\
\hline \hline
\end{tabular}

is the time spent during steps $2-4$ of the $\mathrm{PE}(\mathrm{CE})^{m}$ scheme, and finally $T_{\text {tot }}^{\mathrm{imp}}$ and $T_{\mathrm{tot}}^{\exp }$ are the total time-marching times required by implicit and explicit MOT solvers, respectively. In Table I, $\left\{N_{\text {iter }}^{\text {imp }} F_{\text {iter }}^{\text {imp }}\right\}_{\text {avg }}$ and $m_{\text {avg }}$ represent the average values of $N_{\text {iter }}^{\text {imp }} F_{\text {iter }}^{\text {imp }}$ and $m$ computed over all $N_{\mathrm{t}}$ steps, respectively.

As expected, $T_{\text {fix }}^{\text {imp }}$ and $T_{\text {fix }}^{\text {exp }}$ are almost the same. At low frequencies (i.e., large $\Delta t$ ), the $T_{\mathrm{PECE}}^{\mathrm{exp}}<T_{\mathrm{TFQMR}}^{\mathrm{imp}}$ because $m_{\text {avg }}<\left\{N_{\text {iter }}^{\mathrm{imp}} F_{\text {iter }}^{\mathrm{imp}}\right\}_{\text {avg }}$. For these values of $\Delta t, T_{\mathrm{PECE}}^{\exp }$ is almost one third of $T_{\mathrm{TFQMR}}^{\mathrm{imp}}$; and since $T_{\mathrm{PECE}}^{\exp } \gg T_{\text {fix }}^{\exp }$ and $T_{\mathrm{TFQMR}}^{\mathrm{imp}} \gg T_{\text {fix }}^{\mathrm{imp}}$, the explicit MOT solver is more than two times faster than the implicit MOT solver. For high frequency excitations (i.e., small $\Delta t$ ), both solvers require almost the same time. The measurement results provided in Table I verify the statements about computational complexities of the solvers given in Sec. II E.

Table II compares $\sigma_{\text {err }}^{\operatorname{imp}}\left(f_{0}\right)$ and $\sigma_{\text {err }}^{\exp }\left(f_{0}\right)$, and it shows that the explicit MOT solver is slightly more accurate than its implicit counterpart (which uses the same $\Delta t$ ) in all the simulations. The table also shows that when the number of unknowns is increased from $N_{\mathrm{s}}=696$ to $N_{\mathrm{s}}=2376$, the accuracy increases for the same $f_{0}$ (and also the same $\Delta t$ ). However, when the number of unknowns is increased from $N_{\mathrm{s}}=2376$ to $N_{\mathrm{s}}=6756$, there is no change in the accuracy.

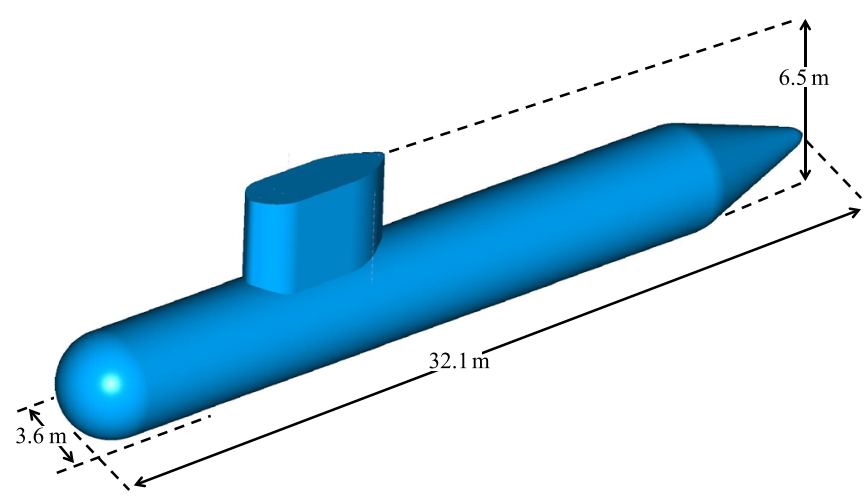

FIG. 4. (Color online) Geometry and dimensions of the submarine.

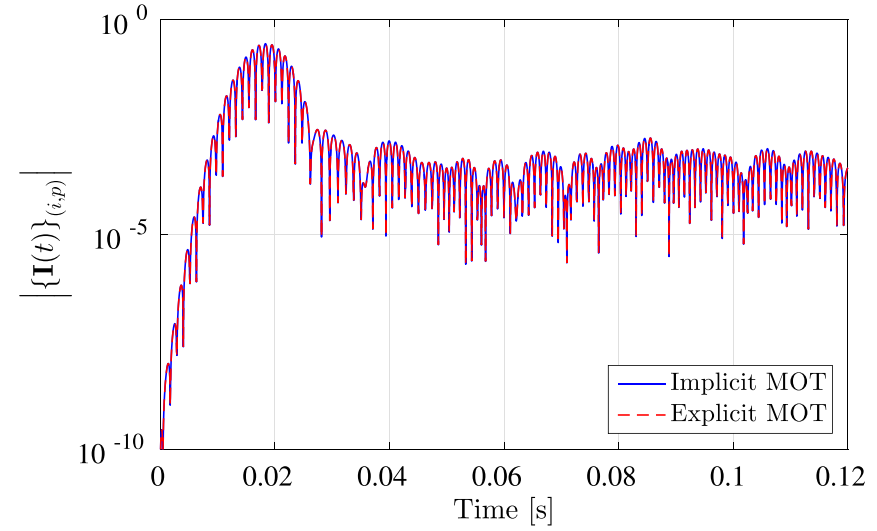

FIG. 5. (Color online) Comparison of $\left|\{\mathbf{I}(t)\}_{(i, p)}\right|, p=260, i=1$, computed by the implicit and explicit MOT solvers at $\mathbf{r}_{(i, p)}=(2.431,0.715,4.183) \mathrm{m}$.

This can simply be explained by the fact that for these spatial discretization levels, the accuracy is bounded by the temporal discretization and can, for example, be increased by reducing $\Delta t$ and/or increasing $P$.

\section{B. Submarine}

For this example, transient acoustic scattering from a rigid submarine is analyzed using the implicit and explicit

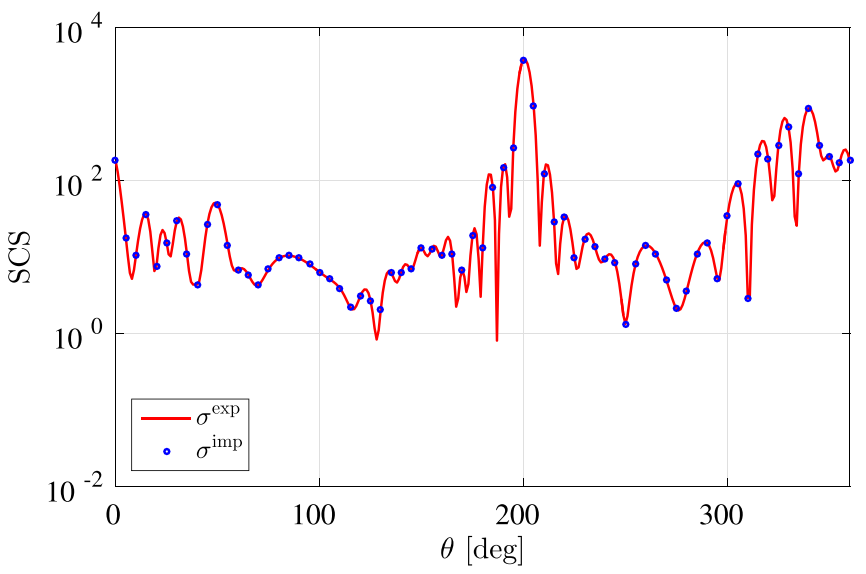

(a)

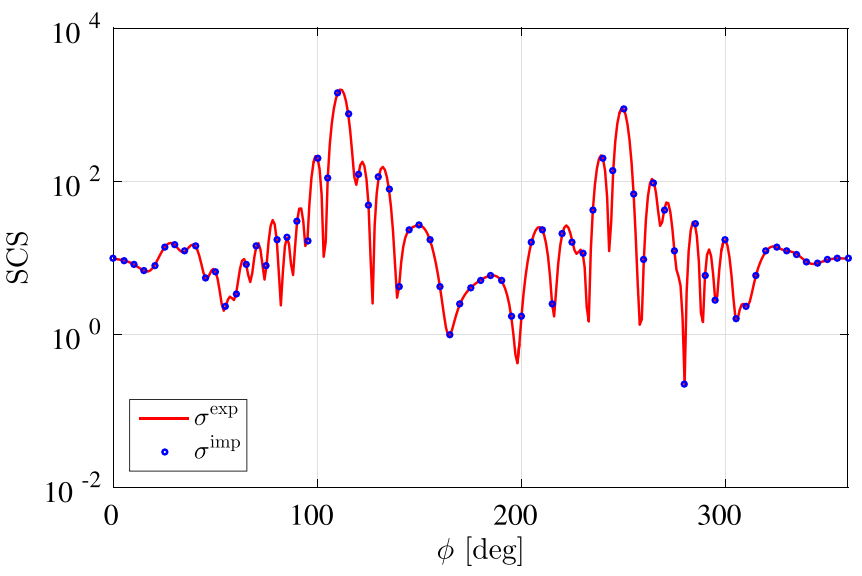

(b)

FIG. 6. (Color online) Comparison of SCS values obtained at $f=f_{0}$ on the (a) $x z$-plane (i.e., $\theta=\left[0^{\circ}, 360^{\circ}\right]$ and $\phi=0^{\circ}$ ) and (b) $x y$-plane (i.e., $\theta=90^{\circ}$ and $\left.\phi=\left[0^{\circ}, 360^{\circ}\right]\right)$ from the solutions computed by the implicit and explicit MOT schemes. 


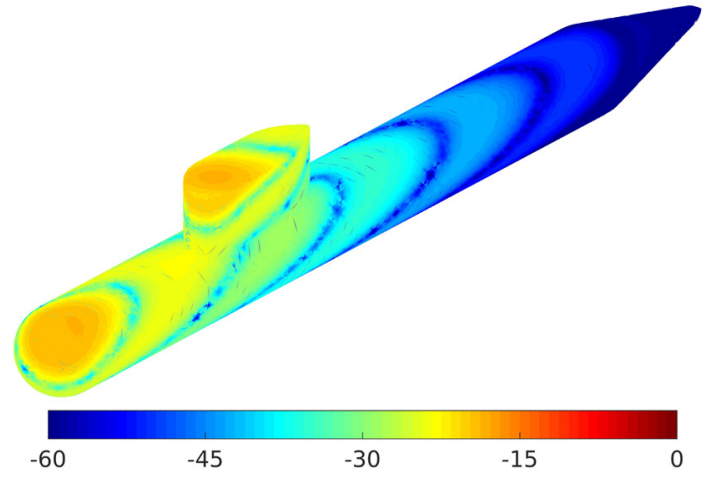

(a)

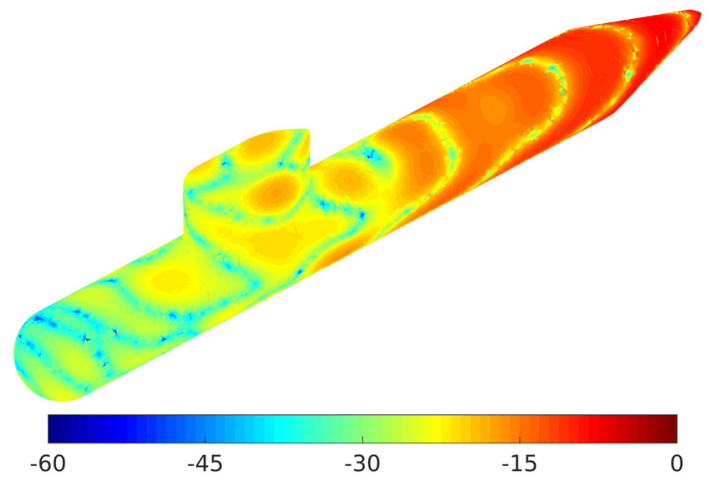

(c)

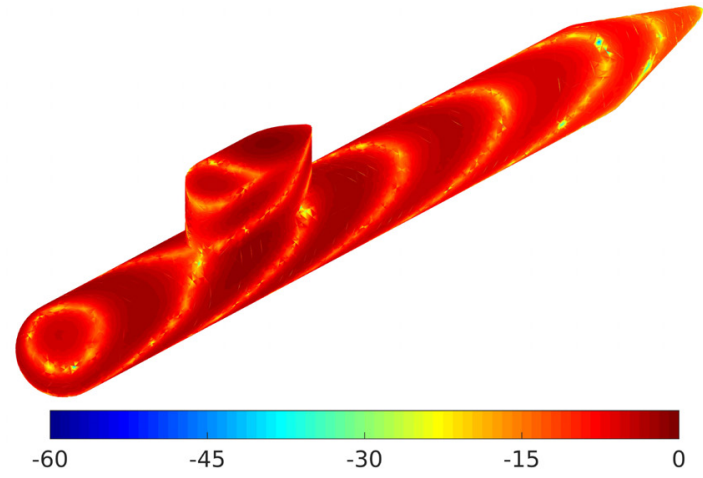

(b)

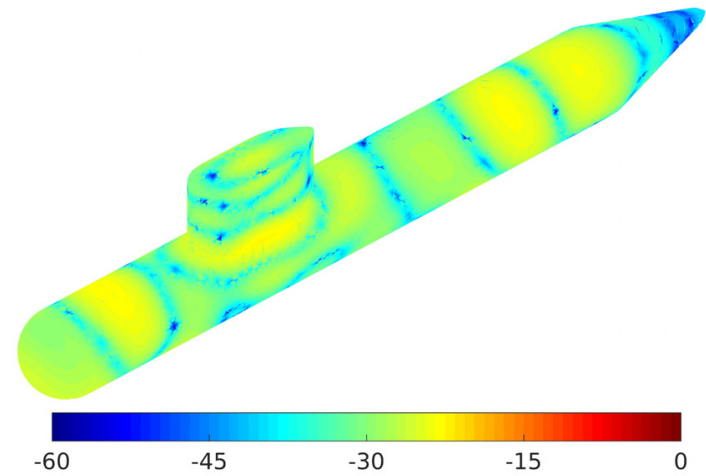

(d)

FIG. 7. (Color online) Snapshots of the velocity potential on the surface of the submarine at times: (a) $150 \Delta t$; (b) $300 \Delta t$; (c) $450 \Delta t$, and (d) $800 \Delta t$.

MOT solvers. The submarine fits in a box of dimensions $32.1 \times 3.6 \times 6.5 \mathrm{~m}$ as shown in Fig. 4, Its surface is discretized into $N_{\mathrm{p}}=3422$ curvilinear triangular patches resulting in $N_{\mathrm{s}}=20532$ number of unknowns. The temporal interpolation function $T(t)$ is the third-order Lagrange polynomial, i.e., $P=3$. For the explicit MOT solver, the SOR coefficient $\alpha=1$ (i.e., no SOR is applied) and $\mathbf{p}$ stores the coefficients of the Adams-Bashforth method and c stores the coefficients of the backward difference method resulting in a $\mathrm{PE}(\mathrm{CE})^{m}$ scheme with $K=4 .{ }^{38}$ Excitation parameters $\hat{\mathbf{k}}^{\mathrm{i}}=(-0.354,-0.354,-0.866)$ (arrival angle of the plane wave is defined using $\theta=150^{\circ}$ and $\phi=225^{\circ}$ ), $f_{0}=437.25 \mathrm{~Hz}, f_{\mathrm{bw}}=163.96 \mathrm{~Hz}, w=2.912 \mathrm{~ms}$, and $t_{0}$ $=17.47 \mathrm{~ms}$; and the simulations are carried out for $N_{\mathrm{t}}=1840$ times steps with $\Delta t=0.0653 \mathrm{~ms}$.

Figure 5 compares the $\left|\{\mathbf{I}(t)\}_{(i, p)}\right|, p=260, i=1$, computed by the implicit and explicit MOT solvers at $\mathbf{r}_{(i, p)}=(2.431,0.715,4.183) \mathrm{m}$. The solutions agree very well. Figures 6(a) and 6(b) provide the SCS values obtained at $f=f_{0}$ on the $x z$ - plane (i.e., $\theta=\left[0^{\circ}, 360^{\circ}\right]$ and $\phi=0^{\circ}$ ) and the $x y$ - plane (i.e., $\theta=90^{\circ}$ and $\phi=\left[0^{\circ}, 360^{\circ}\right]$ ) from the solutions computed by the implicit and explicit MOT schemes. The results are in very good agreement. Snapshots of the velocity potential on the surface of the submarine at times $150 \Delta t, 300 \Delta t, 450 \Delta t$, and $800 \Delta t$ are shown in Figs. 7(a)-7(d).

\section{CONCLUSION}

A fully explicit MOT scheme to solve the TDKIE for analyzing transient acoustic scattering from rigid objects is developed. The velocity potential on the surface of the scatterer is expanded using a higher-order polynomials in space. Inserting this expansion in the TDKIE and point-testing the resulting equation (i.e., Nyström method in space) yield a system of ODEs. This system is integrated in time for the unknown expansion coefficients using a $\mathrm{PE}(\mathrm{CE})^{m}$ method. The resulting time marching scheme does not call for matrix equation solution since the Gram matrix resulting from the point-testing of the polynomials is diagonal. Numerical results demonstrate that the proposed explicit MOT scheme is significantly faster than its implicit counterpart under low frequency excitation (i.e., for large time steps) while maintaining the accuracy and the stability of the solution.

Extension of the proposed explicit solver, which could be used in the solution of resonance-free Burton-Miller surface integral equation, is underway.

\section{ACKNOWLEDGMENTS}

This publication is based upon work supported by the King Abdullah University of Science and Technology (KAUST) Office of Sponsored Research (OSR) under Award No 2016-CRG5-2953. The authors would like to 
thank the King Abdullah University of Science and Technology Supercomputing Laboratory (KSL) for providing the required computational resources.

\section{APPENDIX: NUMERICAL EVALUATION OF MATRIX ELEMENTS}

The evaluation of the first term in the right hand side of Eq. (8) is rather straightforward and its value depends only on $\vartheta^{-1}\left(\mathbf{r}_{(j, q)}\right)$ and $\left.\partial_{t} T(t)\right|_{t=(h-l) \Delta t}$.

The second term in the right hand side of Eq. (8) is equal to $\left\{\mathbf{Z}_{h-l}^{\exp }\right\}_{(j, q)(i, p)}$ and is evaluated as described next.

When patches $q$ and $p$ are not within close proximity of each other (e.g., they are more than two patches apart), the evaluation of $\left\{\mathbf{Z}_{h-l}^{\exp }\right\}_{(j, q)(i, p)}$ in Eq. (15) is carried out numerically using a Gaussian quadrature,

$$
\begin{aligned}
\left\{\mathbf{Z}_{h-l}^{\exp }\right\}_{(j, q)(i, p)}= & -\omega_{(i, p)} \hat{\mathbf{n}}^{\prime}\left(\mathbf{r}_{(i, p)}\right) \cdot \frac{\mathbf{R}}{8 \pi R^{3}} \\
& \times\left[\partial_{t} T(t)+\frac{R}{c_{0}} \partial_{t}^{2} T(t)\right]_{t=(h-l) \Delta t-R / c_{0}},
\end{aligned}
$$

where $\mathbf{R}=\mathbf{r}_{(j, q)}-\mathbf{r}_{(i, p)}, R=|\mathbf{R}|$, and $\omega_{(i, p)}$ is the quadrature weight at the interpolation point $\mathbf{r}_{(i, p)}$. Note that $\mathbf{r}_{(i, p)}$ exactly coincides with the Gaussian quadrature points. ${ }^{33}$ Also, note that $\mathbf{r}_{(i, p)}$ (and Gaussian quadrature points) are generated on the rightangle triangle in the $(\xi, \eta)$ space and mapped back to Cartesian coordinate system where the curvilinear triangular patch resides.

When patches $q$ and $p$ are not within close proximity of each other but do not overlap, a higher degree Gaussian quadrature is used to evaluate the integral,

$$
\begin{aligned}
\left\{\mathbf{Z}_{h-l}^{\exp }\right\}_{(j, q)(i, p)}= & -\sum_{\nu=1}^{N_{\mathrm{v}}} \omega_{\nu} L_{(i, p)}\left(\mathbf{r}_{\nu}\right) \hat{\mathbf{n}}^{\prime}\left(\mathbf{r}_{\nu}\right) \cdot \frac{\mathbf{R}}{8 \pi R^{3}} \\
& \times\left[\partial_{t} T(t)+\frac{R}{c_{0}} \partial_{t}^{2} T(t)\right]_{t=(h-l) \Delta t-R / c_{0}},
\end{aligned}
$$

where $\mathbf{R}=\mathbf{r}_{(j, q)}-\mathbf{r}_{\nu}, R=|\mathbf{R}|, \omega_{\nu}$ is the quadrature weight at the quadrature point $\mathbf{r}_{\nu}$, and $N_{\nu}$ is the number of Gaussian quadrature points. Note that the term $L_{(i, p)}\left(\mathbf{r}_{\nu}\right)$ is needed since $\mathbf{r}_{\nu}$ and $\mathbf{r}_{(i, p)}$ do not overlap. Also note that, like before, these quadrature points are generated on the right-angle triangle in the $(\xi, \eta)$ space and mapped back to Cartesian coordinate system as $\mathbf{r}_{\nu}$.

When patches $q$ and $p$ overlap, the integrand becomes singular. In this case, the integral is evaluated in two parts,

$$
\begin{aligned}
\left\{\mathbf{Z}_{h-l}^{\exp }\right\}_{(j, q)(i, p)}= & -\int_{S_{p}} \vartheta^{-1}\left(\mathbf{r}^{\prime}\right) L_{(i, p)}\left(\mathbf{r}^{\prime}\right) \hat{\mathbf{n}}^{\prime}\left(\mathbf{r}^{\prime}\right) \cdot \frac{\mathbf{R}}{4 \pi c_{0} R^{2}} \\
& \times\left[\partial_{t}^{2} T(t)\right]_{t=(h-l) \Delta t-R / c_{0}} d s^{\prime} \\
& -\int_{S_{p}} \vartheta^{-1}\left(\mathbf{r}^{\prime}\right) L_{(i, p)}\left(\mathbf{r}^{\prime}\right) \hat{\mathbf{n}}^{\prime}\left(\mathbf{r}^{\prime}\right) \cdot \frac{\mathbf{R}}{4 \pi R^{3}} \\
& \times\left[\partial_{t} T(t)\right]_{t=(h-l) \Delta t-R / c_{0}} d s^{\prime} \\
= & I_{1}+I_{2} .
\end{aligned}
$$

The first integral $I_{1}$ has a singularity of $1 / R$ and is evaluated after Duffy transformation is applied. ${ }^{47}$ The second integral $I_{2}$ has a singularity of $1 / R^{2}$. To account for this singularity, the integral in the $(\xi, \eta)$ space is expressed in terms of $\rho$ and $\theta$, variables of the local polar coordinate system centered at $\mathbf{r}_{(\xi, \eta)}\left[\mathbf{r}_{(j, q)} \text { transformed into the }(\xi, \eta) \text { space] (Fig. 8) }\right)^{48}$

$$
\begin{aligned}
I_{2} & =-\int_{S_{p}} \vartheta^{-1}\left(\mathbf{r}^{\prime}\right) L_{(i, p)}\left(\mathbf{r}^{\prime}\right) \hat{\mathbf{n}}^{\prime}\left(\mathbf{r}^{\prime}\right) \cdot \frac{\mathbf{R}}{4 \pi R^{3}}\left[\partial_{t} T(t)\right]_{t=(h-l) \Delta t-R / c_{0}} d s^{\prime}=-\int_{0}^{1} \int_{0}^{1-\xi} L_{(i, p)}\left(\mathbf{r}^{\prime}\right) \hat{\mathbf{n}}^{\prime}\left(\mathbf{r}^{\prime}\right) \cdot \frac{\mathbf{R}}{4 \pi R^{3}}\left[\partial_{t} T(t)\right]_{t=(h-l) \Delta t-R / c_{0}} d \eta d \xi \\
& =\lim _{\varepsilon \rightarrow 0} \int_{0}^{2 \pi} \int_{\varepsilon}^{\rho(\theta)} \underbrace{-\rho L_{(i, p)}\left(\mathbf{r}^{\prime}\right) \hat{\mathbf{n}}^{\prime}\left(\mathbf{r}^{\prime}\right) \cdot \frac{\mathbf{R}}{4 \pi R^{3}}\left[\partial_{t} T(t)\right]_{t=(h-l) \Delta t-R / c_{0}}}_{F(\rho, \theta)} d \rho d \theta
\end{aligned}
$$

Here, $d s^{\prime}=\vartheta\left(\mathbf{r}^{\prime}\right) d \xi d \eta, d \xi d \eta=\rho d \rho d \theta, \rho(\theta)$ is the distance between $\mathbf{r}_{(\xi, \eta)}$ and the boundary of the right-angle triangle along the direction of $\theta$ [Fig. 8(b)]. $F(\rho, \theta)$ in Eq. (A4) has a singularity of $1 / \rho$, which is "extracted" as described below. ${ }^{48} F(\rho, \theta)$ can be expressed as

$$
F(\rho, \theta)=\frac{F_{-1}(\theta)}{\rho}+O(1),
$$

where $F_{-1}(\theta)$ is a real function of $\theta$, which satisfies $\int_{0}^{2 \pi} F_{-1}(\theta) d \theta=0$. The first term of the right hand side of Eq. (A5) is subtracted and added (after being integrated) back to $I_{2}$ (Ref. 48),

$$
\begin{aligned}
I_{2}= & \lim _{\varepsilon \rightarrow 0} \int_{0}^{2 \pi} \int_{\varepsilon}^{\rho(\theta)}\left[F(\rho, \theta)-\frac{F_{-1}(\theta)}{\rho}\right] d \rho d \theta+\lim _{\varepsilon \rightarrow 0} \int_{0}^{2 \pi} \int_{\varepsilon}^{\rho(\theta)}\left[\frac{F_{-1}(\theta)}{\rho}\right] d \rho d \theta=\int_{0}^{2 \pi} \int_{0}^{\rho(\theta)}\left[F(\rho, \theta)-\frac{F_{-1}(\theta)}{\rho}\right] d \rho d \theta \\
& +\lim _{\varepsilon \rightarrow 0} \int_{0}^{2 \pi} F_{-1}(\theta) \ln \rho(\theta) d \theta-\lim _{\varepsilon \rightarrow 0}\{\ln \varepsilon \underbrace{\int_{0}^{2 \pi} F_{-1}(\theta) d \theta}_{0}\}=\int_{0}^{2 \pi} \int_{0}^{\rho(\theta)}\left[F(\rho, \theta)-\frac{F_{-1}(\theta)}{\rho}\right] d \rho d \theta+\int_{0}^{2 \pi} F_{-1}(\theta) \ln \rho(\theta) d \theta .
\end{aligned}
$$

All the integrands in Eq. (A6) are regular and are numerically evaluated using a Gauss-Legendre quadrature. 


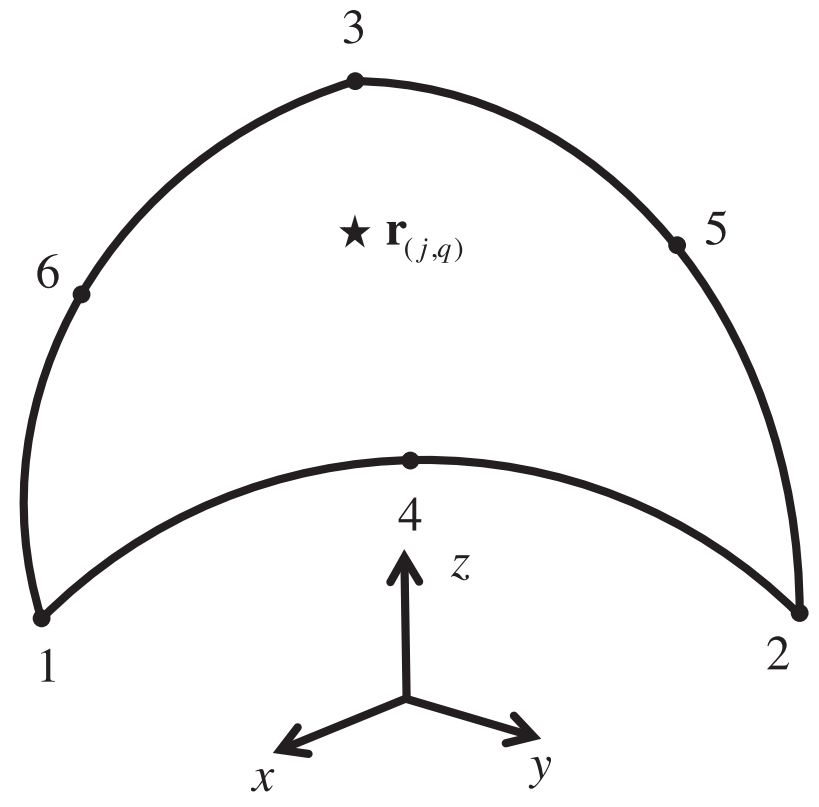

(a)

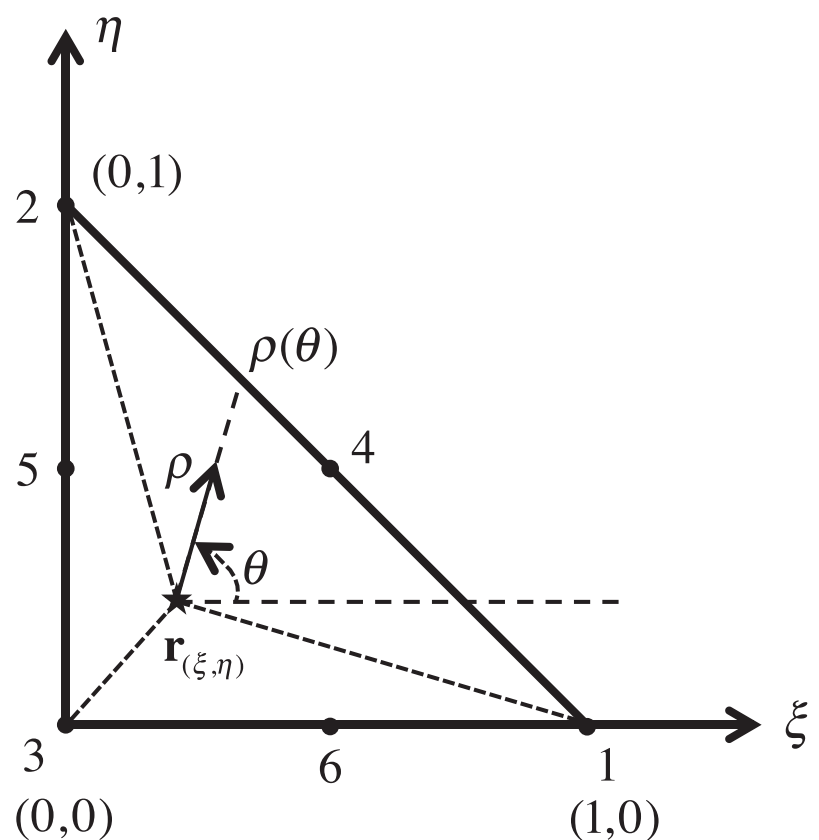

(b)

FIG. 8. (a) Interpolation point $\mathbf{r}_{(j, q)}$ in the Cartesian coordinate system. (b) Interpolation point $\mathbf{r}_{(\xi, \eta)}\left(\mathbf{r}_{(j, q)}\right.$ transformed into the $(\xi, \eta)$ space) and the local polar coordinate system defined in $(\xi, \eta)$ space.

${ }^{1} \mathrm{~S}$. Wang, "Finite-difference time-domain approach to underwater acoustic scattering problems," J. Acoust. Soc. Am. 99(4), 1924-1931 (1996).

${ }^{2}$ K. Manoj and S. Bhattacharyya, "Transient acoustic radiation from impulsively accelerated bodies by the finite element method," J. Acoust. Soc. Am. 107(3), 1179-1188 (2000).

${ }^{3}$ J.-M. Jin, The Finite Element Method in Electromagnetics (Wiley-IEEE, Hoboken, NJ, 2014).

${ }^{4}$ J.-M. Jin, Theory and Computation of Electromagnetic Fields (WileyIEEE, Hoboken, NJ, 2015).

${ }^{5}$ M. J. Bluck and S. P. Walker, "Analysis of three-dimensional transient acoustic wave propagation using the boundary integral equation method," Int. J. Numer. Methods Eng. 39(8), 1419-1431 (1996).
${ }^{6}$ S. Dodson, S. Walker, and M. Bluck, "Implicitness and stability of time domain integral equation scattering analyses," Appl. Comput. Electromagn. Soc. J. 13, 291-301 (1998).

${ }^{7}$ M. Bluck and S. Walker, "Time-domain BIE analysis of large threedimensional electromagnetic scattering problems," IEEE Trans. Antennas Propag. 45(5), 894-901 (1997).

${ }^{8}$ Y. Ding, A. Forestier, and T. H. Duong, "A Galerkin scheme for the time domain integral equation of acoustic scattering from a hard surface," J. Acoust. Soc. Am. 86, 1566-1572 (1989).

${ }^{9}$ T. Ha-Duong, B. Ludwig, and I. Terrasse, "A Galerkin BEM for transient acoustic scattering by an absorbing obstacle," Int. J. Numer. Methods Eng. 57(13), 1845-1882 (2003).

${ }^{10}$ A. Ergin, B. Shanker, and E. Michielssen, "Analysis of transient wave scattering from rigid bodies using a Burton-Miller approach," J. Acoust. Soc. Am. 106(5), 2396-2404 (1999).

${ }^{11}$ A. Ergin, B. Shanker, and E. Michielssen, "Fast transient analysis of acoustic wave scattering from rigid bodies using a two-level plane wave time domain algorithm," J. Acoust. Soc. Am. 106(5), 2405-2416 (1999).

${ }^{12}$ A. Ergin, B. Shanker, and E. Michielssen, "Fast analysis of transient acoustic wave scattering from rigid bodies using the multilevel plane wave time domain algorithm," J. Acoust. Soc. Am. 107(3), 1168-1178 (2000).

${ }^{13}$ B. Rynne, "Stability and convergence of time marching methods in scattering problems," IMA J. Appl. Math. 35(3), 297-310 (1985).

${ }^{14}$ P. J. Davies, "Numerical stability and convergence of approximations of retarded potential integral equations," SIAM J. Numer. Anal. 31(3), 856-875 (1994).

${ }^{15}$ D. S. Weile, G. Pisharody, N.-W. Chen, B. Shanker, and E. Michielssen, "A novel scheme for the solution of the time-domain integral equations of electromagnetics," IEEE Trans. Antennas Propag. 52(1), 283-295 (2004).

${ }^{16}$ Y. Beghein, K. Cools, H. Bagci, and D. De Zutter, "A space-time mixed Galerkin marching-on-in-time scheme for the time-domain combined field integral equation," IEEE Trans. Antennas Propag. 61(3), 1228-1238 (2013).

${ }^{17}$ A. J. Pray, Y. Beghein, N. V. Nair, K. Cools, H. Bagci, and B. Shanker, "A higher order space-time Galerkin scheme for time domain integral equations," IEEE Trans. Antennas Propag. 62(12), 6183-6191 (2014).

${ }^{18}$ A. Pray, N. Nair, and B. Shanker, "Stability properties of the time domain electric field integral equation using a separable approximation for the convolution with the retarded potential," IEEE Trans. Antennas Propag. 60(8), 3772-3781 (2012).

${ }^{19}$ B. Shanker, M. Lu, J. Yuan, and E. Michielssen, "Time domain integral equation analysis of scattering from composite bodies via exact evaluation of radiation fields," IEEE Trans. Antennas Propag. 57(5), 1506-1520 (2009).

${ }^{20}$ Y. Shi, M.-Y. Xia, R.-S. Chen, E. Michielssen, and M. Lu, "Stable electric field TDIE solvers via quasi-exact evaluation of MOT matrix elements," IEEE Trans. Antennas Propag. 59(2), 574-585 (2011).

${ }^{21}$ B. Shanker, A. A. Ergin, K. Aygun, and E. Michielssen, "Analysis of transient electromagnetic scattering phenomena using a two-level plane wave time-domain algorithm," IEEE Trans. Antennas Propag. 48(4), 510-523 (2000).

${ }^{22}$ B. Shanker, A. A. Ergin, M. Lu, and E. Michielssen, "Fast analysis of transient electromagnetic scattering phenomena using the multilevel plane wave time domain algorithm," IEEE Trans. Antennas Propag. 51(3), 628-641 (2003).

${ }^{23}$ A. E. Yilmaz, D. S. Weile, B. Shanker, J.-M. Jin, and E. Michielssen, "Fast analysis of transient scattering in lossy media," IEEE Antennas Wireless Propag. Lett. 1(1), 14-17 (2002).

${ }^{24}$ A. E. Yilmaz, J.-M. Jin, and E. Michielssen, "Time domain adaptive integral method for surface integral equations," IEEE Trans. Antennas Propag. 52(10), 2692-2708 (2004).

${ }^{25} \mathrm{H}$. Bagci, A. E. Yilmaz, V. Lomakin, and E. Michielssen, "Fast solution of mixed-potential time-domain integral equations for half-space environments," IEEE Trans. Geosci. Remote Sens. 43(2), 269-279 (2005).

${ }^{26} \mathrm{H}$. Bagci, A. E. Yilmaz, J.-M. Jin, and E. Michielssen, "Fast and rigorous analysis of EMC/EMI phenomena on electrically large and complex cable-loaded structures," IEEE Trans. Electromagn. Compat. 49(2), 361-381 (2007).

${ }^{27}$ H. A. Ülkü, H. Bagci, and E. Michielssen, "Marching on-in-time solution of the time domain magnetic field integral equation using a predictorcorrector scheme," IEEE Trans. Antennas Propag. 61(8), 4120-4131 (2013). 
${ }^{28}$ S. Rao, D. Wilton, and A. Glisson, "Electromagnetic scattering by surfaces of arbitrary shape," IEEE Trans. Antennas Propag. 30(3), 409-418 (1982).

${ }^{29}$ A. D. Pierce, Acoustics: An Introduction to its Physical Principles and Applications (McGraw-Hill, New York, 1981).

${ }^{30}$ J. Li, D. Dault, and B. Shanker, "A quasianalytical time domain solution for scattering from a homogeneous sphere," J. Acoust. Soc. Am. 135(4), 1676-1685 (2014).

${ }^{31}$ L. F. Canino, J. J. Ottusch, M. A. Stalzer, J. L. Visher, and S. M. Wandzura, "Numerical solution of the Helmholtz equation in 2D and 3D using a high-order Nyström discretization," J. Comput. Phys. 146(2), 627-663 (1998).

${ }^{32}$ M. S. Tong, W. C. Chew, and M. J. White, "Multilevel fast multipole algorithm for acoustic wave scattering by truncated ground with trenches," J. Acoust. Soc. Am. 123(5), 2513-2521 (2008).

${ }^{33}$ G. Kang, J. Song, W. C. Chew, K. C. Donepudi, and J.-M. Jin, "A novel grid-robust higher order vector basis function for the method of moments," IEEE Trans. Antennas Propag. 49(6), 908-915 (2001).

${ }^{34}$ G. Manara, A. Monorchio, and R. Reggiannini, "A space-time discretization criterion for a stable time-marching solution of the electric field integral equation," IEEE Trans. Antennas Propag. 45(3), 527-532 (1997).

${ }^{35}$ K. Aygun, B. Shanker, A. A. Ergin, and E. Michielssen, "A two-level plane wave time-domain algorithm for fast analysis of EMC/EMI problems," IEEE Trans. Electromagn. Compat. 44(1), 152-164 (2002).

${ }^{36} \mathrm{R}$. W. Freund, "A transpose-free quasi-minimal residual algorithm for non-Hermitian linear systems," SIAM J. Sci. Comput. 14(2), 470-482 (1993).

${ }^{37}$ S. B. Sayed, H. Ulku, and H. Bagci, "Stable quasi-explicit MOT solver for the time domain volume electric field integral equation," in Applied Computational Electromagnetics Symposium (2014), pp. 416-420.
${ }^{38}$ E. Hairer, S. P. Norsett, and G. Warner, Solving Ordinary Differential Equations I: Nonstiff Problems (Springer, New York, 2010).

${ }^{39}$ A. Glaser and V. Rokhlin, "A new class of highly accurate solvers for ordinary differential equations,” J. Sci. Comput. 38(3), 368-399 (2009).

${ }^{40}$ A. Dutt, L. Greengard, and V. Rokhlin, "Spectral deferred correction methods for ordinary differential equations," BIT Numer. Math. 40(2), 241-266 (2000)

${ }^{41}$ D. Colton and R. Kress, Integral Equation Methods in Scattering Theory (SIAM, Philadelphia, PA, 2013).

${ }^{42}$ Y. Shi, H. Bagci, and M. Lu, "On the internal resonant modes in marching-on-in-time solution of the time domain electric field integral equation,” IEEE Trans. Antennas Propag. 61(8), 4389-4392 (2013).

${ }^{43}$ Y. Shi, H. Bagci, and M. Lu, "On the DC loop modes in the MOT solution of the time domain EFIE," in IEEE Antennas and Propagation Society Symposium (2014), pp. 1891-1892.

${ }^{44}$ C. C. Chien, H. Rajiyah, and S. N. Atluri, "An effective method for solving the hyper-singular integral equations in 3-D acoustics," J. Acoust. Soc. Am. 88(2), 918-937 (1990).

${ }^{45}$ Y. Brick and A. Boag, "Multilevel nonuniform grid algorithm for acceleration of integral equation-based solvers for acoustic scattering," IEEE Trans. Ultrason. Ferroelectr. Freq. Control 57(1), 262-273 (2010).

${ }^{46}$ S. Turley, "Acoustic scattering from a sphere," Class Notes, Department of Physics and Astronomy, Brigham Young University, Provo, UT (2006).

${ }^{47}$ M. G. Duffy, "Quadrature over a pyramid or cube of integrands with a singularity at a vertex," SIAM J. Numer. Anal. 19(6), 1260-1262 (1982).

${ }^{48}$ M. Guiggiani, G. Krishnasamy, T. J. Rudolphi, and F. Rizzo, "A general algorithm for the numerical solution of hypersingular boundary integral equations," J. Appl. Mech. 59(3), 604-614 (1992). 